\title{
ASYMMETRIC SIMPLE EXCLUSION PROCESS WITH OPEN BOUNDARIES AND KOORNWINDER POLYNOMIALS
}

\author{
LUIGI CANTINI
}

\begin{abstract}
In this paper we analyze the steady state of the Asymmetric Simple Exclusion process with open boundaries and second class particles by deforming it through the introduction of spectral parameters. The (unnormalized) probabilities of the particle configurations get promoted to Laurent polynomials in the spectral parameters and are constructed in terms of non-symmetric Koornwinder polynomials. In particular we show that the partition function coincides with a symmetric Macdonald-Koornwinder polynomial. As an outcome we compute the steady current and the average density of first class particles.
\end{abstract}

Date: August 14, 2018.

The work of LC is partially supported by CNRS through a "Chaire d'excellence". It is a pleasure to thank Jan de Gier for collaboration at an erly stage of this project and the Departement of Mathematics and Statistics of the University of Melbourne for kind hospitality. 


\section{INTRODUCTION}

The Asymmetric Simple Exclusion Process (ASEP) is a paradigmatic example of an out of equilibrium systems [36, 16, 9, 17]. In its simplest form the ASEP consists of particles located on the sites of a directed one dimensional lattice under the condition that each site can be occupied by at most one particle, so that the local configurations on each site can be denoted by a $\bullet$ for an occupied site and by a $\circ$ for an empty site. The particles are subject to a stochastic evolution which consists of jumps of a particle on an empty neighboring sites with rates $t^{\frac{1}{2}}$ or $t^{-\frac{1}{2}}$ depending on the direction of the jump.

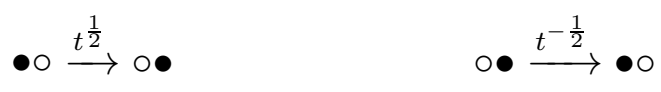

Despite its apparent simplicity, this model can be successfully employed to describe or at least to capture the main features of very different physical systems. Indeed the ASEP has appeared for the first time in biology [29] but since then it has found applications in the study of a wide range of physical phenomena: traffic flow, surface growth, sequence alignment, etc. (see [9] for a recent review of many of these applications).

The ASEP can be in particular used to model the exchange of particles between two reservoirs at different chemical potential [17. In this case one considers a lattice of finite length $N$ and the particles can be exchanged with a left and a right reservoir [33]. So, besides a rate for right and left jumps of a particle, we have also rates $\alpha, \gamma$, respectively for a particle injected in or removed from the leftmost site and $\delta, \beta$ for a particle injected in or removed from the rightmost site.

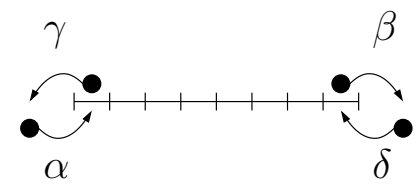

Apart for its physical interest, this model has recently drawn the attention also of combinatorialists. Indeed the (unnormalized) stationary probability of the particle configurations are given by enumerations of certain classes of tableaux [21, 11, 12].

The model we are going to consider here has as an extra feature, the presence of second class particles, which are confined in the finite lattice, i.e. that are not exchanged with the reservoirs at the boundaries. These second class particles can be thought as some sort of mobile impurities and will be indicated by $*$. Their jump rates on empty sites, 
or exchange rates with usual (first class) particles are given by

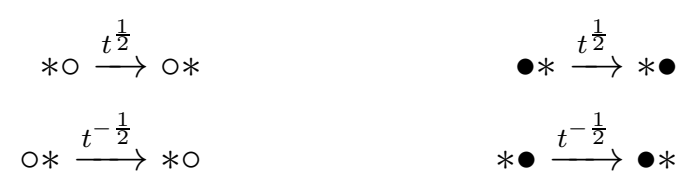

We shall call this model for short "open 2ASEP".

The Markov chain of the 2ASEP turns out to be integrable for a generic choice of the parameters introduced above. Actually it is not difficult to show that the ASEP (in absence of second class particles) is algebraically equivalent to a spin $\frac{1}{2}$ chain of XXZ type with non diagonal boundaries. A great deal of effort has been devoted to exploit the integrable structure of the ASEP, either through Bethe ansatz techniques or separation of variables or other powerfull methods of the theory of integrable systems, in order to study the full spectrum of the ASEP Markov matrix. Reviewing all these studies goes beyond the scopes of the present paper, the interested reader can have a look at the nice introduction of [27] and references therein.

A different approach, which goes under the name of Matrix Product Ansatz [18], has proven to be more successful in computing the properties of the asymptotic steady state of the system [3]. This approach consists in writing the stationary probability of any particles configuration as a trace of products of some auxiliary (infinite dimensional) matrices that satisfy a certain algebra. For concrete computations one has to find manageable representations of the matrix algebra.

In absence of second class particles, Uchiyama, Sasamoto and Wadaty [40] have provided a representation of the Matrix algebra in terms of the Askey-Wilson polynomials, which allows for the exact computation at finite size of the steady state current and of its the density. These quantities are given by rather simple expression involving the moments of the Askey-Wilson measure. Combining these results of [40] with a remarkable combinatorial expression for the stationary measure of particle configurations in terms of staircase tableaux [12], Corteel and Williams have provided a very interesting combinatorial formula for the moments of the Askey-Wilson polynomials [12] (see also [10]).

In [39], Uchiyama has tackled the case in presence of second class particles, using a representation of the matrix algebra in terms of a t-boson. He was able to compute the so called "partition function" as a certain contour integral and from it to compute the phase diagram of the model in the thermodynamic limit. Nonetheless his finite size formulas are quite complicated if compared with the one obtained in 
absence of second class particles in [40] using the Askey-Wilson polynomials.

In the present paper we put forward an approach to the computation of the steady state of the open 2ASEP that bypasses the Matrix Product Ansatz. Such an approach has been pioneered by Di Francesco and Zinn-Justin in the context of the stochastic dense $O(1)$ loop model [19] and is based just on the integrability of the model. In this introduction we are going to present just the rough idea behind this approach, whose details are discussed in the body of the paper.

Given our choice of jump rates we know that the open 2ASEP is integrable, which means in particular that its Markov matrix $\mathcal{M}$ can be obtained starting from the so called scattering matrices $\mathcal{S}_{i}$. These matrices will be defined in Section 2 , here we only remark that they depend on a set of spectral parameters $\mathbf{z}=\left\{z_{1}, \ldots, z_{N}\right\}$, where $N$ is length of the lattice and the the parameter $z_{i}$ is "attached" the $i$-th site. The vector $\Psi_{N, m}$, gathering the stationary probabilities of each 2ASEP configurations on a system of length $N$ in the sector with $m$ second class particles, can be obtained by the eigenvector $\Psi_{N, m}(\mathbf{z})$ of the scattering matrices

$$
\mathcal{S}_{i}(\mathbf{z}) \Psi_{N, m}(\mathbf{z})=\Psi_{N, m}(\mathbf{z})
$$

upon specialization $z_{i}=1$

$$
\Psi_{N, m}=\Psi_{N, m}(\mathbf{1}) .
$$

The advantage of dealing with $\Psi_{N, m}(\mathbf{z})$ is that, this vector can be normalized in such a way that it is a Laurent polynomial in the variables $\mathbf{z}$ and it satisfies a set of exchange-reflection relations that correspond to a degeneration of the quantum Knizhnik-Zamolodchikov (qKZ) equations of type $\tilde{\mathcal{C}}_{N}$ (see [24, 34 for some recent results on polynomial solutions of qKZ equations of type $\tilde{\mathcal{C}}_{N}$ ). We shall construct the solution of the qKZ equations using non-symmetric Koornwinder polynomials and study some of their properties (notice the recent work 38] on the relations between Koornwinder polynomials and the XXZ spin chain). We shall prove that the normalization of $\Psi_{N, m}(\mathbf{z})$ (sometimes called the "partition function") is given by a symmetric Macdonald-Koornwinder polynomial for a single column partition of length $N-m$. From the weighted partition function we shall extract current and average density of first class particles. Finally, using a known integral representation for the Macdonald-Koornwinder polynomials we shall compute these quantities in the thermodynamic limit, in the case of a fixed density of second class particles, recovering the phase diagram of [39]. 
The paper is organized as follows. In Section 2 we shall discuss the integrability of the open 2ASEP. In particular we explain how to make to model inhomogeneous by introducing the spectral parameters $\mathbf{z}$ and show in Theorem 1 that the inhomogeneous generalization of the stationary probability $\Psi(\mathbf{z})$ satisfies a set of exchange-reflection relations. The algebraic structures behind these equations will be discussed in Appendix A, where we also recall some basic facts about symmetric Macdonald-Koornwinder polynomials, non-symmetric Koornwinder polynomials and Askey-Wilson polynomials. In Section 3 we analyze the solution of the qKZ equations. We show in Theorems 3, 4 that the normalization of $\Psi(\mathbf{z})$ and the generating function for the total density of first class particles are given by symmetric Macdonald-Koornwinder polynomials. In Section 4 we shall discuss some recursion relations between solutions of the exchange-reflection equations for systems of different size and different values of the parameters $a, b, c, d$. In Section 5 we compute the average current and density of first class particles, we shall then take the thermodynamic limit and compute the phase diagram for the model.

\section{INTEGRABILITY AND EXCHANGE-REFLECTION EQUATIONS}

The Markov matrix which generates the time evolution of the 2ASEP with second class particles can be written as a sum of local terms. Let us associate to each site a vector space $V \simeq \mathbb{C}^{3}$ with basis vectors $\left\{v_{-1}, v_{0}, v_{1}\right\}$ which correspond to the following local particle configurations

$$
\begin{aligned}
v_{-1} \longleftrightarrow \circ & \text { empty site } \\
v_{0} \longleftrightarrow * & \text { second class particle } \\
v_{1} \longleftrightarrow \bullet & \text { first class particle }
\end{aligned}
$$

The vector space with a basis labeled by global particle configurations has a tensor product structure $H_{N}=V_{1} \otimes V_{2} \otimes \cdots \otimes V_{N}$, with $V_{i} \simeq$ $V \simeq \mathbb{C}^{3}$. The local contributions to the Markov chain are given by the following operators $\mathbf{e} \in \operatorname{End}(V \otimes V), \mathbf{f}(\rho, \sigma) \in \operatorname{End}(V)$

$$
\begin{aligned}
& \mathbf{e}=\sum_{i, j=-1}^{1}\left(E^{(i, j)} \otimes E^{(j, i)}-E^{(j, j)} \otimes E^{(i, i)}\right) t^{\frac{1}{2} \operatorname{sign}(j-i)} \\
& \mathbf{f}(\rho, \sigma)=\rho\left(E^{(-1,1)}-E^{(1,1)}\right)+\sigma\left(E^{(1,-1)}-E^{(-1,-1)}\right)
\end{aligned}
$$


where the operators $E^{(j, i)} \in \operatorname{End}(V)$ are defined by $E^{(j, i)} v_{k}=\delta_{j, k} v_{i}$. The Markov matrix $\mathcal{M}$ reads

$$
\mathcal{M}=\sum_{i=1}^{N-1} \mathbf{e}_{i}+\mathbf{f}_{1}(\gamma, \alpha)+\mathbf{f}_{N}(\beta, \delta) .
$$

where

$$
\begin{aligned}
\mathbf{e}_{i} & =\mathbf{1}_{1} \otimes \cdots \otimes \mathbf{1}_{i-1} \otimes \mathbf{e} \otimes \mathbf{1}_{i+2} \otimes \cdots \otimes \mathbf{1}_{N} \\
\mathbf{f}_{i}(\rho, \sigma) & =\mathbf{1}_{1} \otimes \cdots \otimes \mathbf{1}_{i-1} \otimes \mathbf{f}(\rho, \sigma) \otimes \mathbf{1}_{i+1} \otimes \cdots \otimes \mathbf{1}_{N}
\end{aligned}
$$

Since the dynamics preserves the number of second class particles, we have a splitting of $H_{N}$ as a direct sum

$$
H_{N}=\bigoplus_{m=0}^{N} H_{N, m}
$$

of subspaces (sectors) $H_{N, m}$ with fixed number $m$ of second class particles, which are preserved by the action of $\mathcal{M}$.

A first glimpse of the algebraic structure behind the Markov matrix $\mathcal{M}$ comes from the observation that the operators $\mathbf{e}_{i}, \mathbf{f}_{1}(\gamma, \alpha)$ and $\mathbf{f}_{N}(\beta, \delta)$ generate a representation of $\mathcal{H}_{N}$, the affine Hecke algebra of type $\hat{\mathcal{C}}_{N}$. Indeed, the operators $T_{0}, T_{1}, \ldots, T_{N}$ defined as

$$
\begin{aligned}
& T_{0}=\alpha^{-\frac{1}{2}} \gamma^{-\frac{1}{2}} \mathbf{f}_{1}(\gamma, \alpha)+\alpha^{\frac{1}{2}} \gamma^{-\frac{1}{2}} \mathbf{1} \\
& T_{N}=\beta^{-\frac{1}{2}} \delta^{-\frac{1}{2}} \mathbf{f}_{N}(\beta, \delta)+\beta^{\frac{1}{2}} \delta^{-\frac{1}{2}} \mathbf{1}
\end{aligned}
$$

and for $1 \leq i \leq N-1$

$$
T_{i}=\mathbf{e}_{i}+t^{-\frac{1}{2}} \mathbf{1}
$$

satisfy the commutation relations of the generators of $\mathcal{H}_{N}$ (see Section 3.1.)

$$
\begin{aligned}
T_{i}-T_{i}^{-1} & =t_{i}^{\frac{1}{2}}-t_{i}^{-\frac{1}{2}} \\
T_{i} T_{j} & =T_{j} T_{i} \text { if }|i-j|>1 \\
T_{i} T_{i+1} T_{i} & =T_{i+1} T_{i} T_{i+1} \text { if } i \neq 0, N-1 \\
T_{0} T_{1} T_{0} T_{1} & =T_{1} T_{0} T_{1} T_{0} \\
T_{N} T_{N-1} T_{N} T_{N-1} & =T_{N-1} T_{N} T_{N-1} T_{N}
\end{aligned}
$$

with $t_{0}^{\frac{1}{2}}=\alpha^{\frac{1}{2}} \gamma^{-\frac{1}{2}}, t_{N}^{\frac{1}{2}}=\beta^{\frac{1}{2}} \delta^{-\frac{1}{2}}$ and $t_{i}=t$ for $1 \leq i \leq N-1$.

Operators written as sums of generators of $\mathcal{H}_{N}$, like $\mathcal{M}$ are known to be quantum integrable [35, 20]. In what follows we shall present only a few notions of the theory of quantum integrable systems needed in order to study the properties of the stationary measure. 
The first ingredient are the $\check{R}$ matrices based on the baxterization of the Hecke algebra of type $A_{n}$, which read

$$
\check{R}_{i}(z)=\mathbf{1}+\frac{z-1}{t^{\frac{1}{2}} z-t^{-\frac{1}{2}}} \mathbf{e}_{i}
$$

They satisfy the braided Yang-Baxter equation (YBE)

$$
\check{R}_{i}\left(y z^{-1}\right) \check{R}_{i+1}\left(x z^{-1}\right) \check{R}_{i}\left(x y^{-1}\right)=\check{R}_{i+1}\left(x y^{-1}\right) \check{R}_{i}\left(x z^{-1}\right) \check{R}_{i+1}\left(y z^{-1}\right)
$$

and the so called unitarity condition

$$
\check{R}_{i}(z) \check{R}_{i}\left(z^{-1}\right)=1
$$

The second ingredient are the boundary scattering matrices. A classification of all the integrable boundary scattering matrices compatible with the matrices $\check{R}_{i}(z)$ has been obtained recently [14], here we shall consider the case that corresponds to the baxterization of the boundary matrices $\mathbf{f}_{1}(\delta, \alpha)$ and $\mathbf{f}_{N}(\beta, \gamma)$. For $t \neq 1$ it turns out to be convenient to parametrize the boundary rates as

$$
\begin{array}{ll}
\alpha=\frac{\left(t^{\frac{1}{2}}-t^{-\frac{1}{2}}\right) a b}{(a-1)(b-1)}, & \gamma=\frac{t^{-\frac{1}{2}}-t^{\frac{1}{2}}}{(a-1)(b-1)} \\
\beta=\frac{\left(t^{\frac{1}{2}}-t^{-\frac{1}{2}}\right) c d}{(c-1)(d-1)}, & \delta=\frac{t^{-\frac{1}{2}}-t \frac{1}{2}}{(c-1)(d-1)}
\end{array}
$$

This means that for $t>1$ we can choose $a, c<0$ and $b, d>1$, while for $0<t<1$ we have $a, c<0$ and $0<b, d<1$. The boundary scattering matrices read

$$
\begin{gathered}
K_{1}(z \mid a, b)=\mathbf{1}+\frac{\left(z^{2}-1\right)}{(z-a)(z-b)} \gamma^{-1} \mathbf{f}_{1}(\gamma, \alpha), \\
K_{N}(z \mid c, d)=\mathbf{1}+\frac{\left(1-z^{2}\right)}{(c z-1)(d z-1)} \delta^{-1} \mathbf{f}_{N}(\beta, \delta) .
\end{gathered}
$$

They satisfy the boundary Yang-Baxter equations (BYBE), also called reflection equations [35, 8]

$$
\begin{aligned}
&\left.\check{R}_{1}\left(x y^{-1}\right) K_{1}(y) \check{R}_{1}\left(x^{-1} y^{-1}\right)\right) K_{1}(x)= \\
& K_{1}(x) \check{R}_{1}\left(x^{-1} y^{-1}\right) K_{1}(y) \check{R}_{1}\left(x y^{-1}\right), \\
& \check{R}_{N-1}\left(x y^{-1}\right) K_{N}(x) \check{R}_{N-1}(x y) K_{N}(y)= \\
& K_{N}(y) \check{R}_{N-1}(x y) K_{N}(x) \check{R}_{N-1}\left(x y^{-1}\right)
\end{aligned}
$$

and the unitarity conditions

$$
K_{1}(x) K_{1}\left(x^{-1}\right)=\mathbf{1}, \quad K_{N}(x) K_{N}\left(x^{-1}\right)=\mathbf{1} .
$$


Here is the explicit form of the $K$ matrices acting respectively on the first and last site

$$
K_{1}(z \mid a, b)=\left(\begin{array}{ccc}
1 & 0 & 0 \\
0 & 1 & 0 \\
0 & 0 & 1
\end{array}\right)+\frac{z^{2}-1}{(z-a)(z-b)}\left(\begin{array}{ccc}
-1 & 0 & -a b \\
0 & 0 & 0 \\
1 & 0 & a b
\end{array}\right),
$$

$$
K_{R}(z \mid c, d)=\left(\begin{array}{ccc}
1 & 0 & 0 \\
0 & 1 & 0 \\
0 & 0 & 1
\end{array}\right)+\frac{1-z^{2}}{(c z-1)(d z-1)}\left(\begin{array}{ccc}
c d & 0 & 1 \\
0 & 0 & 0 \\
-c d & 0 & -1
\end{array}\right) .
$$

The most common way to exploit the algebraic properties of the $\check{R}$ and $K$ matrices is to use such matrices as building blocks of the so called double row transfer matrix, which depends on a spectral parameter $y$ and which commute with the matrix $\mathcal{M}$ for any values of $y$. The diagonalization of $\mathcal{M}$ is then turned into the diagonalization of the transfer matrix [35].

Here instead we take a slightly different approach, which will work effectively for the the determination of the stationary state of $\mathcal{M}$, and is analogous to the one already employed in [5], for the stochastic dense $O(1)$ loop model with open boundaries. Instead of using double row the transfer matrix, we use the so called Scattering Matrices which are defined by

$$
\begin{gathered}
\mathcal{S}_{i}(\mathbf{z})=\check{R}_{i-1}\left(z_{i} z_{i-1}^{-1}\right) \cdots \check{R}_{2}\left(z_{i} z_{2}^{-1}\right) \check{R}_{1}\left(z_{i} z_{1}^{-1}\right) \\
K_{1}\left(z_{i}^{-1}\right) \check{R}_{1}\left(z_{i} z_{1}\right) \cdots \check{R}_{i-2}\left(z_{i} z_{i-2}\right) \check{R}_{i-1}\left(z_{i} z_{i-1}\right) \\
\check{R}_{i}\left(z_{i} z_{i+1}\right) \cdots \check{R}_{N-2}\left(z_{i} z_{N-1}\right) \check{R}_{N-1}\left(z_{i} z_{N}\right) \\
K_{N}\left(z_{i}\right) \check{R}_{N-1}\left(z_{i} / z_{N}\right) \cdots \check{R}_{i+1}\left(z_{i} / z_{i+2}\right) \check{R}_{i}\left(z_{i} / z_{i+1}\right)
\end{gathered}
$$

where $\mathbf{z}=\left\{z_{1}, z_{2}, \ldots, z_{N}\right\}$. Even though the matrices $\mathcal{S}_{i}(\mathbf{z})$ do not commute with $\mathcal{M}$, they commute among themselves

$$
\left[\mathcal{S}_{i}(\mathbf{z}), \mathcal{S}_{j}(\mathbf{z})\right]=0,
$$

and in the limit $\mathbf{z} \rightarrow \mathbf{1}$ their eigenvectors coincide with the eigenvectors of $\mathcal{M}$. Indeed, let $\Psi(\mathbf{z})$ be a common eigenvector of $\mathcal{S}_{i}(\mathbf{z})$, with eigenvalues $\Lambda_{i}(\mathbf{z})$

$$
\mathcal{S}_{i}(\mathbf{z}) \Psi(\mathbf{z})=\Lambda_{i}(\mathbf{z}) \Psi(\mathbf{z})
$$

Call $\mathcal{S}_{i}(z):=\mathcal{S}_{i}\left(z_{j \neq i}=1, z_{i}=z\right), \Lambda(z)=\Lambda\left(z_{j \neq i}=1, z_{i}=z\right)$ and $\Psi_{i}(z):=\Psi\left(z_{j \neq i}=1, z_{i}=z\right)$, so that the specialization $z_{j \neq i}=1$ and $z_{i}=z$ of eqs.(17) reads

$$
\mathcal{S}_{i}(z) \Psi_{i}(z)=\Lambda_{i}(z) \Psi_{i}(z) .
$$


Differentiating this equation with respect to $z$ and then setting $z=1$ one gets

$$
\mathcal{S}_{i}^{\prime}(1) \Psi_{i}(1)+\mathcal{S}_{i}(1) \Psi_{i}^{\prime}(1)=\Lambda_{i}(1) \Psi_{i}^{\prime}(1)+\Lambda_{i}^{\prime}(1) \Psi_{i}(1)
$$

but $\Psi_{i}(1)=\Psi(\mathbf{1}), \mathcal{S}_{i}(1)=\mathbf{1}, \Lambda_{i}(1)=1$ and it s simple to check that

$$
\mathcal{S}_{i}^{\prime}(1)=\frac{2}{t^{\frac{1}{2}}-t^{-\frac{1}{2}}} \mathcal{M}
$$

In this way we have turned the problem of the diagonalization of $\mathcal{M}$ into the simultaneous diagonalization of the Scattering Matrices $\mathcal{S}_{i}(\mathbf{z})$.

The common eigenvectors of $\mathcal{S}_{i}(\mathbf{z})$ have nice covariance properties under the action of the $\check{R}$ and $K$ matrices. Let $\Psi(\mathbf{z})$ be a common eigenvector of $\mathcal{S}_{i}(\mathbf{z})$ with eigenvalues $\Lambda_{i}(\mathbf{z})$ and define

$$
\begin{aligned}
\Psi_{i}(\mathbf{z}) & :=\check{R}_{i}\left(z_{i} z_{i+1}^{-1}\right) \Psi(\mathbf{z}), \\
\tilde{\Psi}_{1}(\mathbf{z}) & :=K_{1}\left(z_{1}\right) \Psi(\mathbf{z}), \\
\tilde{\Psi}_{N}(\mathbf{z}) & :=K_{N}\left(z_{N}\right) \Psi(\mathbf{z}) .
\end{aligned}
$$

The vectors $\Psi_{i}(\mathbf{z}), \tilde{\Psi}_{1}(\mathbf{z})$ and $\tilde{\Psi}_{N}(\mathbf{z})$ are common eigenvectors respectively of

$$
\mathbf{s}_{i} \mathcal{S}_{j}(\mathbf{z}), \quad \mathbf{s}_{0} \mathcal{S}_{i}(\mathbf{z}) \quad \text { and } \quad \mathbf{s}_{N} \mathcal{S}_{i}(\mathbf{z}),
$$

where $\mathbf{s}_{i}$ for $i \neq 0, N$ acts on functions of $\mathbf{z}$ by exchanging $z_{i} \leftrightarrow z_{i+1}$, while $\mathbf{s}_{0}, \mathbf{s}_{N}$ exchange respectively $z_{1} \leftrightarrow z_{1}^{-1}, z_{N} \leftrightarrow z_{N}^{-1}$.

In particular

$$
\begin{aligned}
\left(\mathbf{s}_{i} \mathcal{S}_{j}(\mathbf{z})\right) \Psi_{i}(\mathbf{z}) & =\Lambda_{j}(\mathbf{z}) \Psi_{i}(\mathbf{z}) \quad \text { for } \quad j \neq i, i+1 \\
\left(\mathbf{s}_{i} \mathcal{S}_{i}(\mathbf{z})\right) \Psi_{i}(\mathbf{z}) & =\Lambda_{i+1}(\mathbf{z}) \Psi_{i}(\mathbf{z}) \\
\left(\mathbf{s}_{i} \mathcal{S}_{i+1}(\mathbf{z})\right) \Psi_{i}(\mathbf{z}) & =\Lambda_{i}(\mathbf{z}) \Psi_{i}(\mathbf{z}) \\
\left(\mathbf{s}_{0} \mathcal{S}_{j}(\mathbf{z})\right) \tilde{\Psi}_{1}(\mathbf{z}) & =\Lambda_{j}(\mathbf{z}) \tilde{\Psi}_{i}(\mathbf{z}) \quad \text { for } \quad j \neq 1 \\
\left(\mathbf{s}_{0} \mathcal{S}_{1}(\mathbf{z})\right) \tilde{\Psi}_{1}(\mathbf{z}) & =\Lambda_{1}^{-1}(\mathbf{z}) \tilde{\Psi}_{i}(\mathbf{z}) \\
\left(\mathbf{s}_{N} \mathcal{S}_{j}(\mathbf{z})\right) \tilde{\Psi}_{N}(\mathbf{z}) & =\Lambda_{j}(\mathbf{z}) \tilde{\Psi}_{N}(\mathbf{z}) \quad \text { for } \quad j \neq N \\
\left(\mathbf{s}_{N} \mathcal{S}_{N}(\mathbf{z})\right) \tilde{\Psi}_{N}(\mathbf{z}) & =\Lambda_{N}^{-1}(\mathbf{z}) \tilde{\Psi}_{N}(\mathbf{z})
\end{aligned}
$$

Eqs.(19,21) are consequences of the following commutation relations that are immediate to verify using the YBE, BYBE and unitarity equations

$$
\begin{aligned}
\check{R}_{i}\left(z_{i} z_{i+1}^{-1}\right) \mathcal{S}_{j}(\mathbf{z}) & =\left(\mathbf{s}_{i} \mathcal{S}_{j}(\mathbf{z})\right) \check{R}_{i}\left(z_{i} z_{i+1}^{-1}\right) \quad \text { for } \quad j \neq i, i+1 \\
\check{R}_{i}\left(z_{i} z_{i+1}^{-1}\right) \mathcal{S}_{i}(\mathbf{z}) & =\left(\mathbf{s}_{i} \mathcal{S}_{i+1}(\mathbf{z})\right) \check{R}_{i}\left(z_{i} z_{i+1}^{-1}\right) \\
\check{R}_{i}\left(z_{i} z_{i+1}^{-1}\right) \mathcal{S}_{i+1}(\mathbf{z}) & =\left(\mathbf{s}_{i} \mathcal{S}_{i}(\mathbf{z})\right) \check{R}_{i}\left(z_{i} z_{i+1}^{-1}\right)
\end{aligned}
$$


ASEP WITH OPEN BOUNDARIES AND KOORNWINDER POLYNOMIALS 9

$$
\begin{aligned}
K_{1}\left(z_{1}\right) \mathcal{S}_{j}(\mathbf{z}) & =\left(\mathbf{s}_{0} \mathcal{S}_{j}(\mathbf{z})\right) \quad \text { for } \quad j \neq 1 \\
K_{1}\left(z_{1}\right) \mathcal{S}_{1}(\mathbf{z}) & =\left(\mathbf{s}_{0} \mathcal{S}_{1}^{-1}(\mathbf{z})\right) \\
K_{N}\left(z_{N}\right) \mathcal{S}_{j}(\mathbf{z}) & =\left(\mathbf{s}_{N} \mathcal{S}_{j}(\mathbf{z})\right) \\
K_{N}\left(z_{N}\right) \mathcal{S}_{N}(\mathbf{z}) & =\left(\mathbf{s}_{0} \mathcal{S}_{N}^{-1}(\mathbf{z})\right)
\end{aligned}
$$

Since the $\check{R}$ and $K$ matrices are stochastic and preserve the subspace $H_{N, m}$, the stationary state in this sector is lifted to $\Psi_{N, m}(\mathbf{z})$, the unique solution of the following equation on $H_{N, m}$

$$
\mathcal{S}_{i}(\mathbf{z}) \Psi_{N, m}(\mathbf{z})=\Psi_{N, m}(\mathbf{z}) .
$$

Though such equations are still to difficult to deal with, they tell us that $\Psi_{N, m}(\mathbf{z})$ can be normalized to be polynomial in the spectral parameters z. To really get a control on the solution of eqs.(25) we need the following

Theorem 1. The solution $\Psi_{N, m}(\mathbf{z})$ of eqs.(25) can be normalized in such a way that it satisfies the following set of exchange-reflection equations

$$
\begin{aligned}
\check{R}_{i}\left(z_{i} z_{i+1}^{-1}\right) \Psi_{N, m}(\mathbf{z}) & =\mathbf{s}_{i} \Psi_{N, m}(\mathbf{z}) \\
K_{1}\left(z_{1}\right) \Psi_{N, m}(\mathbf{z}) & =\mathbf{s}_{0} \Psi_{N, m}(\mathbf{z}) \\
K_{N}\left(z_{N}\right) \Psi_{N, m}(\mathbf{z}) & =\mathbf{s}_{N} \Psi_{N, m}(\mathbf{z}) .
\end{aligned}
$$

Proof. As we argued above, $\Psi_{N, m}(\mathbf{z})$ can be normalized to be a polynomial in the spectral parameters $\mathbf{z}$ with no overall factor. Call such minimal polynomial solution $\bar{\Psi}_{N, m}(\mathbf{z})$. Using eqs.(19, 21) and the uniqueness of the solution of eqs.(25) we conclude that $\bar{\Psi}_{N, m}(\mathbf{z})$ satisfies eqs. (26) 28), up to a multiplicative factor

$$
\begin{aligned}
\check{R}_{i}\left(z_{i} z_{i+1}^{-1}\right) \bar{\Psi}_{N, m}(\mathbf{z}) & =f_{i}(\mathbf{z}) \mathbf{s}_{i} \bar{\Psi}_{N, m}(\mathbf{z}) \\
K_{1}\left(z_{1}\right) \bar{\Psi}_{N, m}(\mathbf{z}) & =f_{0}(\mathbf{z}) \mathbf{s}_{0} \bar{\Psi}_{N, m}(\mathbf{z}) \\
K_{N}\left(z_{N}\right) \bar{\Psi}_{N, m}(\mathbf{z}) & =f_{N}(\mathbf{z}) \mathbf{s}_{N} \bar{\Psi}_{N, m}(\mathbf{z}) .
\end{aligned}
$$

Using the analytic structure of the right hand side of eqs.(29) and the unitarity conditions, we conclude that the functions $f_{i}(\mathbf{z})$ can take the form

$$
\begin{aligned}
f_{i}(\mathbf{z}) & =(-1)^{\kappa_{i}}\left(\frac{t^{\frac{1}{2}} z_{i+1}-t^{-\frac{1}{2}} z_{i}}{t^{\frac{1}{2}} z_{i}-t^{-\frac{1}{2}} z_{i+1}}\right)^{\epsilon_{i}} \text { for } i \neq 0, N \\
f_{0}(\mathbf{z}) & =(-1)^{\kappa_{0}} z_{1}^{d_{1}}\left(\frac{(1-a z)(1-b z)}{(z-a)(z-b)}\right)^{\epsilon_{0}} \\
f_{N}(\mathbf{z}) & =(-1)^{\kappa_{n}} z_{N}^{d_{N}}\left(\frac{(z-c)(z-d)}{(1-c z)(1-d z)}\right)^{\epsilon_{i}}
\end{aligned}
$$


for some $\kappa_{i}, \epsilon_{i} \in\{0,1\}$ and where $d_{i}$ is the degree of $\bar{\Psi}_{N, m}(\mathbf{z})$ in $z_{i}$. Setting $z_{i}=z_{i+1}$ in the first of the eqs.(29) leads to

$$
\bar{\Psi}_{N, m}(\mathbf{z})_{z_{i}=z_{i+1}}=(-1)^{\kappa_{i}} \bar{\Psi}_{N, m}(\mathbf{z})_{z_{i}=z_{i+1}}
$$

which forces the $\kappa_{i}=0$, otherwise one would find that $\bar{\Psi}_{N, m}(\mathbf{z})$ is divisible by $z_{i}-z_{i+1}$. In the same way setting $z_{1}= \pm 1$ in the second two or $z_{N}= \pm 1$ in third of eqs.(29) one get $\kappa_{0}=\kappa_{N}=0$ and moreover that $d_{1}$ and $d_{N}$ must be even integers.

The last step of the proof consist in combining eqs.(25) and eqs.(29). This leads to $\epsilon_{i}=0$ and $d_{1}=d_{N}=d$, therefore it is immediate to conclude that the Laurent polynomial

$$
\Psi_{N, m}(\mathbf{z}):=\prod_{i=1}^{N} z_{i}^{-d / 2} \bar{\Psi}_{N, m}(\mathbf{z})_{z_{i}=z_{i+1}}
$$

satisfies eqs.(26, 28).

A simple consequence of Theorem 1 is the following uniqueness result

Theorem 2. The system of equations (26-28) admits a Laurent polynomial solution, unique up to a multiplication of a function invariant under the action of $\mathbf{s}_{i}$.

\section{AnAlysis of the SOlution of THE EXCHANGE-REFLECTION EQUATIONS}

In order to analyze the solution of the exchange-reflection equations (26-28) we need to introduce some algebraic background.

\subsection{Affine Hecke algebra of type $\tilde{\mathcal{C}}_{N}$ and Noumi representa-}

tion. The affine Weyl group $\mathcal{W}_{N}$ of type $\tilde{\mathcal{C}}_{N}$ is the group generated by elements $\mathbf{s}_{0}, \mathbf{s}_{1}, \ldots, \mathbf{s}_{N}$ subject to the relations

$$
\begin{aligned}
\mathbf{s}_{i}^{2} & =1 \\
\mathbf{s}_{i} \mathbf{s}_{j} & =\mathbf{s}_{j} \mathbf{s}_{i} \text { if }|i-j|>1 \\
\mathbf{s}_{i} \mathbf{s}_{i+1} \mathbf{s}_{i} & =\mathbf{s}_{i+1} \mathbf{s}_{i} \mathbf{s}_{i+1} \text { if } i \neq 0, N-1 \\
\mathbf{s}_{0} \mathbf{s}_{1} \mathbf{s}_{0} \mathbf{s}_{1} & =\mathbf{s}_{1} \mathbf{s}_{0} \mathbf{s}_{1} \mathbf{s}_{0} \\
\mathbf{s}_{N} \mathbf{s}_{N-1} \mathbf{s}_{N} \mathbf{s}_{N-1} & =\mathbf{s}_{N-1} \mathbf{s}_{N} \mathbf{s}_{N-1} \mathbf{s}_{N}
\end{aligned}
$$

The finite Weyl group $\mathcal{W}_{N}^{0}$ of type $\mathcal{C}_{N}$ is the subgroup of $\mathcal{W}_{N}$ generated by elements $s_{1}, \ldots s_{N}$. The affine Weyl group $\mathcal{W}_{N}$ has a faithful action, 
depending on a parameter $q$, on $\mathbb{C}\left[z_{1}^{ \pm}, \ldots, z_{N}^{ \pm}\right]$, the space of Laurent Polynomials in $N$ variables

$$
\begin{aligned}
& \mathbf{s}_{i} f\left(z_{1}, \ldots, z_{i}, z_{i+1}, \ldots, z_{N}\right)=f\left(z_{1}, \ldots, z_{i+1}, z_{i}, \ldots, z_{N}\right) \\
& \mathbf{s}_{0} f\left(z_{1}, \ldots, z_{i}, z_{i+1}, \ldots, z_{N}\right)=f\left(q z_{1}^{-1}, \ldots, z_{i}, z_{i+1}, \ldots, z_{N}\right) \\
& \mathbf{s}_{N} f\left(z_{1}, \ldots, z_{i}, z_{i+1}, \ldots, z_{N}\right)=f\left(z_{1}, \ldots, z_{i}, z_{i+1}, \ldots, z_{N}^{-1}\right)
\end{aligned}
$$

It has also actions on $\mathbb{Z}^{N}$, parametrized by the level $\ell \in \mathbb{Z}$,

$$
\begin{aligned}
& \mathbf{s}_{i}\left\{\alpha_{1}, \ldots, \alpha_{i}, \alpha_{i+1}, \ldots, \alpha_{N}\right\}=\left\{\alpha_{1}, \ldots, \alpha_{i+1}, \alpha_{i}, \ldots, \alpha_{N}\right\} \\
& \mathbf{s}_{0}\left\{\alpha_{1}, \ldots, \alpha_{i}, \alpha_{i+1}, \ldots, \alpha_{N}\right\}=\left\{\ell-\alpha_{1}, \ldots, \alpha_{i}, \alpha_{i+1}, \ldots, \alpha_{N}\right\} \\
& \mathbf{s}_{N}\left\{\alpha_{1}, \ldots, \alpha_{i}, \alpha_{i+1}, \ldots, \alpha_{N}\right\}=\left\{\alpha_{1}, \ldots, \alpha_{i}, \alpha_{i+1}, \ldots,-\alpha_{N}\right\} .
\end{aligned}
$$

Notice that the action of $\mathcal{W}_{N}^{0}$ on $\mathbb{C}\left[z_{1}^{ \pm}, \ldots, z_{N}^{ \pm}\right]$can be read off from its action on $\mathbb{Z}^{N}$ by looking at monomials, namely for $w \in \mathcal{W}_{N}^{0}$

$$
w \mathbf{z}^{\alpha}=\mathbf{z}^{w \alpha} .
$$

We define two partial orders on $\mathbb{Z}^{N}$. The first is the usual dominance order: $\alpha \leq \beta$ if for $1 \leq j \leq N$ we have $\sum_{i=1}^{j}\left(\alpha_{i}-\beta_{i}\right) \leq 0$. The second order, $\alpha \preceq \beta$, is defined as follows. Call $\alpha^{+}$the unique element in $\mathcal{W}_{N}^{0} \alpha$ such that $\alpha^{+}$is a partition i.e. such that $\alpha_{i} \geq \alpha_{i+1} \geq 0$. Then we say that $\alpha \preceq \beta$ if $\alpha^{+}<\beta^{+}$or in case $\alpha^{+}=\beta^{+}$if $\alpha \leq \beta$.

The Affine Hecke algebra $\mathcal{H}_{N}$ of type $\tilde{\mathcal{C}}_{N}$ is a deformation of the group algebra of $\mathcal{W}_{N}$, which depends on three parameters $t_{0}, t_{N}, t$ and is generated by elements $T_{0}, T_{1}, \ldots, T_{N}$ subject to the commutations relations

$$
\begin{aligned}
T_{i}-T_{i}^{-1} & =t_{i}^{\frac{1}{2}}-t_{i}^{-\frac{1}{2}} \\
T_{i} T_{j} & =T_{j} T_{i} \text { if }|i-j|>1 \\
T_{i} T_{i+1} T_{i} & =T_{i+1} T_{i} T_{i+1} \text { if } i \neq 0, N-1 \\
T_{0} T_{1} T_{0} T_{1} & =T_{1} T_{0} T_{1} T_{0} \\
T_{N} T_{N-1} T_{N} T_{N-1} & =T_{N-1} T_{N} T_{N-1} T_{N}
\end{aligned}
$$

with $t_{1}=t_{2}=\cdots=t_{N-1}=t$.

The finite Hecke algebra $\mathcal{H}_{N}^{0}$ of type $\mathcal{C}_{N}$ is a sub-algebra of $\mathcal{H}_{N}$ generated by elements $T_{1}, \ldots, T_{N-1}, T_{N}$. It is well known [23] that a basis of $\mathcal{H}_{N}$ is parametrized by elements of $\mathcal{W}_{N}$

$$
T_{w}=T_{i_{1}} T_{i_{2}} \ldots T_{i_{\ell}}
$$

where $w=s_{i_{1}} s_{i_{2}} \ldots s_{i_{\ell}}$ is a reduced expression of $w \in \mathcal{W}_{N}$. 
An important commutative sub-algebra $\mathcal{Y}_{N}$ is generated by Lusztig elements $Y_{1}^{ \pm 1}, \ldots, Y_{N}^{ \pm 1}[28]$

$$
Y_{i}=\left(T_{i} \ldots T_{N-1}\right)\left(T_{N} \ldots T_{0}\right)\left(T_{1}^{-1} \ldots T_{i-1}^{-1}\right) .
$$

In [31], Noumi introduced a representation of $\mathcal{H}_{N}$ depending on 6 parameters $a, b, c, d, t, q$, acting on $\mathbb{C}\left[z_{1}^{ \pm 1}, \ldots, z_{N}^{ \pm 1}\right]$, the space of Laurent polynomials in $N$ variables $\mathbf{z}=\left\{z_{1}, \ldots, z_{N}\right\}$

$$
\begin{aligned}
& \widehat{T}_{i}=t^{\frac{1}{2}}-\left(t^{\frac{1}{2}} z_{i}-t^{-\frac{1}{2}} z_{i+1}\right) \partial_{i} \\
& \widehat{T}_{0}=t_{0}^{\frac{1}{2}}-t_{0}^{-\frac{1}{2}} \frac{\left(z_{1}-a\right)\left(z_{1}-b\right)}{z_{1}} \partial_{0} \\
& \widehat{T}_{N}=t_{N}^{\frac{1}{2}}-t_{N}^{-\frac{1}{2}} \frac{\left(c z_{N}-1\right)\left(d z_{N}-1\right)}{z_{N}} \partial_{N},
\end{aligned}
$$

where $t_{0}=-q^{-1} a b t_{N}=-c d$ and the finite difference operator $\partial_{i}, \partial_{0}$ and $\partial_{N}$ are defined by

$$
\partial_{i}=\frac{1-\mathbf{s}_{i}}{z_{i}-z_{i+1}}, \quad \partial_{0}=\frac{1-\mathbf{s}_{0}}{z_{1}-q z_{1}^{-1}}, \quad \partial_{N}=\frac{1-\mathbf{s}_{N}}{z_{N}-z_{N}^{-1}} .
$$

3.2. Analysis of eqs.(26-28). Let us write the wave function $\Psi_{N, m}(\mathbf{z})$ in the basis $\mathbf{w}=w_{1} \ldots w_{N}, w_{i}=0, *$,

$$
\Psi_{N, m}(\mathbf{z})=\sum_{\mathbf{w} \in \mathcal{Q}(N, m)} \psi_{\mathbf{w}}(\mathbf{z}) \mathbf{w},
$$

where the sum runs on $\mathcal{Q}(N, m)$, the set of 2 ASEP configurations on a strip of length $N$ in the presence of $m$ second class particles. We can identify a word in $\circ, *$, $\bullet$ with an element of $\mathbb{Z}^{N}$ through the rules $\circ \equiv-1, * \equiv 0, \bullet \equiv+1$, hence the set $\mathcal{Q}(N, m)$ inherits an action of $\mathcal{W}_{N}^{0}$ and of $\mathcal{W}_{N}$ at level 0 and the partial orders $\leq, \preceq$.

Once written in components, the exchange-reflection eqs.(26]28) are expressed in a nice compact form using the generators of the affine Hecke algebra $\mathcal{H}_{N}$ in the Noumi representation at $q=1$

$$
\begin{array}{llrl}
\psi_{w}=t_{i}^{-\frac{1}{2}} \widehat{T}_{i} \psi_{w} & \text { if } & w=s_{i} w \\
\psi_{s_{i} w}=t_{i}^{\frac{1}{2}} \widehat{T}_{i} \psi_{w} & \text { if } & \left\{\begin{array}{lll}
i>0 & \& & w<s_{i} w \\
i=0 & \& & w>s_{0} w
\end{array}\right.
\end{array}
$$

Recall that for $1 \leq i \leq N-1, t_{i}=t$, while $t_{0}=-a b$ and $t_{N}=-c d$.

In words, eq. (50) tells us that if $w_{i}=w_{i+1}$ then $\psi_{w}$ is symmetric under exchange $z_{i} \leftrightarrow z_{i+1}$, while if $w_{0}=*$ or $w_{N}=*$, then $\psi_{w}$ is respectively symmetric under inversions $z_{1} \rightarrow z_{1}^{-1}$ or $z_{N} \rightarrow z_{N}^{-1}$. Eq.(51)

\footnotetext{
${ }^{1}$ Here we shall just consider $a, b, c, d, t, q \in \mathbb{C}$.
} 
tells us how to exchange two different neighboring particles

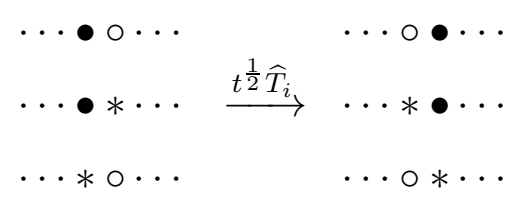

and how to inject/remove particles from the boundaries.

$$
\circ \cdots \stackrel{t_{0}^{\frac{1}{2}} \widehat{T}_{0}}{\longrightarrow} \bullet \cdots \quad \cdots \bullet \stackrel{t_{N}^{\frac{1}{2}} \widehat{T}_{N}}{\longrightarrow} \cdots \circ
$$

3.3. Reference component. Let us consider a system of length $N=$ $k+m$, in the sector with $m$ second class particles, and analyze the component associated to the state

$$
o^{k} *^{m}=\{\underbrace{o \cdots o}_{k} \underbrace{* \cdots * *}_{m}\}
$$

It follows from the eqs. (50151), that $\psi_{\mathrm{o}^{k}{ }^{m}}(\mathbf{z})$ is preserved by the Lusztig operators $Y_{i}$. Therefore (see Appendix A) we can identify $\psi_{\mathrm{o}^{k} *^{m}}(\mathbf{z})$ with the non-symmetric Koornwinder

$$
\psi_{\mathrm{o}_{*} m}(\mathbf{z})=E_{\mu(k, m)}(\mathbf{z})
$$

associated to the string

$$
\mu(k, m)=\{\underbrace{-1, \ldots,-1}_{k}, \underbrace{0, \ldots, 0}_{m}\} .
$$

This fixes completely the normalization of $\Psi_{N, m}(\mathbf{z})$.

The non-symmetric Koornwinder polynomials depend on the further parameter $q$ which appears in the general Noumi representation. Hence a priory we should specify that $E_{\mu(k, m)}(\mathbf{z})$ is taken for $q=1$, but actually we will see in a moment that $E_{\mu(k, m)}(\mathbf{z})$ doesn't depend on $q$.

Moreover, while the monomials expansion of any non-symmetric Koornwinder polynomial reads as in eq.(101), the expansion of $E_{\mu(k, m)}(\mathbf{z})$ is much simpler, it contains only negative powers of $z_{i}$ for $1 \leq i \leq k$.

Either from the exchange eq.(150) applied to $\psi_{\mathrm{o}_{*} m}(\mathbf{z})$ or from the general theory of non-symmetric Koornwinder polynomials [32] we know that $E_{\mu(k, m)}(\mathbf{z})$ is separately symmetric in the first $k$ and last $m$ spectral parameters, so that we can express it as a sum of elementary symmetric polynomials in $z$ variables

$$
E_{\mu(k, m)}(\mathbf{z})=\sum_{i=0}^{k} h_{i}^{(k, m)} e_{k-i}\left(z_{1}^{-1}, \ldots, z_{k}^{-1}\right)
$$


with $h_{0}^{(k, m)}=1$. Using the symmetry properties of $E_{\mu(k, m)}(\mathbf{z})$ we can rewrite its defining equation (102) as

$$
\widehat{T}_{k} \widehat{T}_{k+1} \ldots \widehat{T}_{N-1} \widehat{T}_{N} \widehat{T}_{N-1} \ldots \widehat{T}_{1} \widehat{T}_{0} E_{\mu(k, m)}(\mathbf{z})=E_{\mu(k, m)}(\mathbf{z})
$$

Pictorially this equation has a clear meaning: the action of $\widehat{T}_{0}$ injects a particle on the left, then $\widehat{T}_{N-1} \ldots \widehat{T}_{1}$ moves this particle to the far right, $\widehat{T}_{N}$ removes the particle from the right and finally $\widehat{T}_{k} \widehat{T}_{k+1} \ldots \widehat{T}_{N-1}$ brings back the empty state in position $k$.

Equation (54) allows to determine $h_{j}^{(k, m)}$ in a recursive way. It turns out that they do not depend on $k$ and have a rather simple dependence on $m$. This is the content of the following

Proposition 1. The non symmetric Koornwinder polynomial $E_{\mu(k, m)}(\mathbf{z})$ does not depend on $q$ and reads

$$
E_{\mu(k, m)}(\mathbf{z})=\oint_{0} \frac{d w}{2 \pi i w} H\left(w ; a, b, t^{m} c, t^{m} d\right) \prod_{i=1}^{k}\left(w^{-1}+z_{i}^{-1}\right)
$$

where the integration is around zero and $H(w ; a, b, c, d)$ is a formal power series in $w$

$$
H(w ; a, b, c, d)=\sum_{j=0}^{\infty} h_{n}(a, b, c, d) w^{n}
$$

with

$$
\begin{aligned}
& h_{n}(a, b, c, d)= \\
& \frac{1}{(a b c d ; t)_{n}} \sum_{\substack{0 \leq i, j, k \leq n \\
i+j+k+h \leq n}}\left[\begin{array}{c}
n \\
i, j, k
\end{array}\right]_{t} t^{\left(\begin{array}{c}
i \\
2
\end{array}\right)+\left(\begin{array}{l}
j \\
2
\end{array}\right) a^{i} b^{j}(-c)^{n-k}(-d)^{i+j+k}}
\end{aligned}
$$

where we have used the usual q-multinomial with base $t$

$$
\left[\begin{array}{c}
n \\
i, j, k
\end{array}\right]_{t}=\frac{(t ; t)_{n}}{(t ; t)_{i}(t ; t)_{j}(t ; t)_{k}(t ; t)_{n-i-j-k}}, \quad(a ; t)_{n}=\prod_{i=0}^{n-1}\left(1-a t^{i}\right) .
$$

Proof. Let us rewrite eq.(53) as a contour integral around the origin

$$
E_{\mu(k, m)}(\mathbf{z})=\oint_{0} \frac{d w}{2 \pi i w} H_{k, m}(w) \prod_{i=1}^{k}\left(w^{-1}+z_{i}^{-1}\right)
$$


with $H_{k, m}(w)=\sum_{j=0}^{k} h_{j}^{(k, m)} w^{j}$. Using the following relations

$$
\begin{aligned}
\widehat{T}_{0}\left(w^{-1}+z_{1}^{-1}\right) & =q^{-1} t_{0}^{-\frac{1}{2}}\left(\left(w+z_{1}\right)-\frac{(w+a)(w+b)}{w}\right) \\
\widehat{T}_{j}\left(w+z_{j}\right)\left(w^{-1}+z_{j+1}^{-1}\right) & =t^{-\frac{1}{2}}\left(w^{-1}+z_{j}^{-1}\right)\left(w+z_{j+1}\right) \\
\widehat{T}_{j}\left(w^{-1}+z_{j+1}^{-1}\right) & =t^{-\frac{1}{2}}\left(t w^{-1}+z_{j}^{-1}\right) \\
\widehat{T}_{j}\left(w+z_{j}\right) & =t^{-\frac{1}{2}}\left(t w+z_{j+1}\right) \\
\widehat{T}_{N}\left(w+z_{N}\right) & =t_{N}^{-\frac{1}{2}}\left(\left(w^{-1}+z_{N}^{-1}\right)-\frac{(1+w c)(1+w d)}{w}\right)
\end{aligned}
$$

in eq. (54) we get

$$
\begin{gathered}
t_{N}^{\frac{1}{2}} t^{m} \oint_{0} \frac{d w}{2 \pi i} H_{k, m}(w) \frac{(a+w)(b+w)}{w} \prod_{j=1}^{k-1}\left(t w^{-1}+z_{j}^{-1}\right)= \\
t_{N}^{-\frac{1}{2}} \oint_{0} \frac{d w}{2 \pi i} H_{k, m}(w) \frac{\left(1+t^{m} c w\right)\left(1+t^{m} d w\right)}{w} \prod_{j=1}^{k-1}\left(w^{-1}+z_{j}^{-1}\right)
\end{gathered}
$$

The fact that this equation must be true for any choice of $z_{1}, \ldots, z_{k-1}$ means that we can substitute $\prod_{j}^{k-1}\left(w^{-1}+z_{j}^{-1}\right)$ with any polynomial $p\left(w^{-1}\right)$ of degree $k-1$, getting

$$
\begin{array}{r}
\oint_{0} \frac{d w}{2 \pi i} H_{k, m}(w)\left(p\left(t w^{-1}\right) \frac{(a+w)(b+w)}{w}\right)= \\
\oint_{0} \frac{d w}{2 \pi i} H_{k, m}(w)\left(p\left(w^{-1}\right) \frac{\left(w+t^{-m} c^{-1}\right)\left(w+t^{-m} d^{-1}\right)}{w}\right)
\end{array}
$$

From the previous equation we can already make a couple of conclusions.

1) Since in eq.(59) the parameter $q$ (which was present through $t_{0}=$ $\left.-q^{-1} a b\right)$ has disappeared we have that $E_{\mu(k, m)}(\mathbf{z})$ does not depend on $q$.

2) The dependence on $k$ is only in the degree of the arbitrary polynomial $p(w)$, which means that the coefficients $h_{j}^{(k, m)}$ actually do not depend on $k$, therefore we can suppress the label $k$ and we think of $H_{k, m}(w)=H_{m}(w)$ as a formal infinite series.

3) The parameter $m$ appears just by multiplying both $c$ and $d$ by $t^{m}$ and we can write the solution of eq.(59) as

$$
H_{m}(w ; a, b, c, d)=H\left(w ; a, b, t^{m} c, t^{m} d\right)
$$

and

$$
h_{j}^{(m)}=h_{j}\left(a, b, t^{m} c, t^{m} d\right)
$$


It remains only to use eq.(59) to determine the coefficients $h_{j}(a, b, c, d)$. By setting $p(w)=w^{n-2}$ into eq.(59) we get following three-terms recursion relation

$$
\begin{aligned}
& \left(t^{n-1} a b-c^{-1} d^{-1}\right) h_{n}+ \\
& \quad\left(t^{n-1}(a+b)-\left(c^{-1}+d^{-1}\right)\right) h_{n-1}+\left(t^{n-1}-1\right) h_{n-2}=0 .
\end{aligned}
$$

with initial conditions $h_{0}=1$. It is then not difficult to check that the expression in eq.(57) satisfies this recursion relation.

An immediate consequence of Proposition 1 is that

$$
\psi_{\circ k_{*} m}(\mathbf{z} ; a, b, c, d)=\psi_{\circ^{k}{ }^{m-1}}\left(\mathbf{z}_{\widehat{N}} ; a, b, t c, t d\right) .
$$

where $\mathbf{z}_{\hat{j}}$ means that the variable $z_{j}$ is absent.

The recursion relation for the coefficients $h_{i}$ eq.(60) has some invariance properties. It is invariant under $a \leftrightarrow c^{-1}, b \leftrightarrow d^{-1}, t \leftrightarrow t^{-1}$, from which (writing explicitly the dependence of $h_{n}$ on $t$ as $h_{n}(a, b, c, d \mid t)$ ) we get the duality relation

$$
h_{n}(a, b, c, d \mid t)=h_{n}\left(c^{-1}, d^{-1}, a^{-1}, b^{-1} \mid t^{-1}\right) .
$$

It is also invariant under

$$
a \rightarrow \lambda^{-1} a, \quad b \rightarrow \lambda^{-1} b, \quad c \rightarrow \lambda c, \quad d \rightarrow \lambda d, \quad h_{n} \rightarrow \lambda^{n} h_{n},
$$

which means that $h_{n}(a, b, c, d)$ is homogeneous in $a^{-1}, b^{-1}, c, d$ of degree $n$ and we have

$$
H\left(\lambda^{-1} w ; \lambda^{-1} a, \lambda^{-1} b, \lambda c, \lambda d\right)=H(w ; a, b, c, d) .
$$

Using repeatedly the relation $t^{-\frac{1}{2}} \widehat{T}_{j}^{-1}\left(w^{-1}+z_{j}^{-1}\right)=\left(t^{-1} w^{-1}+z_{j+1}^{-1}\right)$ we obtain easily the components of all the configurations without first class particles. For $J=\left\{j_{1}, \ldots, j_{k}\right\}, 1 \leq j_{1}<j_{2}<\cdots j_{k} \leq N$ call $\circ(J)_{N}$ the configuration with empty space in positions $J$ and second class particles in all the other sites. Then we have

$$
\psi_{\circ(J)_{N}}(\mathbf{z})=\oint_{0} \frac{d w}{2 \pi i w} H\left(w ; a, b, t^{m} c, t^{m} d\right) \prod_{i=1}^{k}\left(t^{i-j_{i}} w^{-1}+z_{j_{i}}^{-1}\right) .
$$

For configurations of the kind $*^{m} \circ^{k}$, combining the previous equation with eq.(63) we obtain the analog of eq.(61)

$$
\psi_{*^{m} \circ k}(\mathbf{z} ; a, b, c, d)=\psi_{*^{m-1} \circ^{k}}\left(\mathbf{z}_{\hat{1}} ; t^{-1} a, t^{-1} b, c, d\right) .
$$


Analogous integral formulas can be written for the components of the configurations without empty sites. For later reference we write explicitly $h_{1}$

$$
h_{1}(a, b, c, d)=\frac{a+b-c^{-1}-d^{-1}}{c^{-1} d^{-1}-a b} .
$$

Another interesting remark is that from a close inspection it turns out that the three-terms recursion (60) is nothing else than the recursion relation for Al-Salam Chihara polynomials in disguise (see eq.(114)) therefore we have also

$$
h_{n}(a, b, c, d)=\frac{(-1)^{n} c^{\frac{n}{2}} d^{\frac{n}{2}}}{(a b c d ; t)_{n}} Q_{n}\left(\frac{1}{2}\left(\sqrt{\frac{c}{d}}+\sqrt{\frac{d}{c}}\right) ; c^{\frac{1}{2}} d^{\frac{1}{2}} a, c^{\frac{1}{2}} d^{\frac{1}{2}} b \mid t\right) .
$$

3.4. Other components and normalization. Once the component $\psi_{\mathrm{o}^{*} m}$ is known, all the others are obtained from it by action of the generators $\widehat{T}_{i}$ using the exchange equations (51). Well, actually it is not necessary to use the full algebra $\mathcal{H}_{N}, \mathcal{H}_{N}^{0}$ being enough. Moreover the state $\psi_{\circ^{k} *^{m}}$ is preserved by the generators $\widehat{T}_{i}$ for $1 \leq i \neq N-m \leq N$. In order to see how to remove this redundancy and to provide a compact formula for any component $\psi_{w}(\mathbf{z})$ we need to concentrate for a moment on the Weyl group $\mathcal{W}_{N}^{0}$ and its action on $\mathcal{Q}(N, m)$. The state $w(N-$ $m, m)$ is preserved by $I:=\mathcal{S}_{N-m} \times \mathcal{W}_{m}^{0}$, which is a parabolic subgroup of $\mathcal{W}_{N}^{0}$ i.e. a subgroup generated by a subset of the generators of $\mathcal{W}_{N}^{0}$. In the present case $I$ is generated by $\mathbf{s}_{i}$ for $1 \leq i \neq N-m \leq N$. The states in $\mathcal{Q}(N, m)$ are in bijection with elements in $\mathcal{W}_{N}^{0} / I$. On the other hand, from the basic properties of parabolic subgroups of a Coxeter group (see [23] Chapter 1) we know that the set $\mathcal{W}^{I}$ defined

$$
\mathcal{W}^{I}=\left\{\mathbf{g} \in \mathcal{W}_{N}^{0} \mid \ell(\mathbf{g s})>\ell(\mathbf{g}), \forall \mathbf{s} \in I\right\} .
$$

is in bijection with classes in the quotient $\mathcal{W}_{N}^{0} / I$, or equivalently, that any classes in $[\mathbf{g}] \in \mathcal{W}_{N}^{0} / I$ contains a unique shortest representative $\mathbf{h} \in \mathcal{W}^{I}$ and therefore any $\mathbf{g} \in \mathcal{W}_{N}^{0}$ can be written in a unique way as a product $\mathbf{g}=\mathbf{h} \mathbf{s}$ with $\mathbf{h} \in \mathcal{W}^{I}$ and $\mathbf{s} \in I$. Moreover, any reduced expression of $\mathbf{g}$ is the product of a reduced expression of $\mathbf{h}$ times a reduced expression of $\mathbf{s}$, which in particular implies that at the level of the Hecke algebra, we have a decomposition

$$
T_{\mathbf{g}}=T_{\mathbf{h}} T_{\mathbf{s}}
$$

with $\mathbf{h} \in W^{I}$ and $\mathbf{s} \in I$, and

$$
\chi\left(T_{\mathbf{g}}\right)=\chi\left(T_{\mathbf{h}}\right) \chi\left(T_{\mathbf{s}}\right) .
$$


The bijection between $W^{I}$ and $\mathcal{Q}(N, m)$ is now obvious

$$
\mathbf{h} \mapsto \mathbf{h} \circ^{k} *^{m} .
$$

Coming back to the components we easily check that for $\mathbf{h} \in W^{I}$ we have

$$
\psi_{\mathbf{h}^{k} *^{m}}(\mathbf{z})=\chi\left(T_{\mathbf{h}}\right) T_{\mathbf{h}} \psi_{\mathrm{o}^{k} *^{m}}(\mathbf{z}) .
$$

It is now easy to prove the following theorem that identify the normalization of $\Psi_{N, m}(\mathbf{z})$ (sometimes called also the partition function) with a Macdonald-Koornwinder polynomial [26]

Theorem 3. Let

$$
Z_{N, m}(\mathbf{z}):=\sum_{w \in \mathcal{Q}(N, m)} \psi_{w}(\mathbf{z})
$$

Then we have

$$
Z_{N, m}(\mathbf{z})=P_{\eta(N, m)}(\mathbf{z}) .
$$

where $P_{\eta(N, m)}(\mathbf{z})$ is the Macdonald-Koornwinder polynomial [26] associated to the partition

$$
\eta(N, m)=\{\underbrace{1, \ldots, 1}_{N-m}, \underbrace{0, \ldots, 0}_{m}\} .
$$

Proof. From [32] (see Theorem 6 in Appendix A) we know that

$$
P_{\eta(N, m)}(\mathbf{z}) \propto \sum_{\mathbf{g} \in \mathcal{W}_{N}^{0}} \chi\left(T_{\mathbf{g}}\right) T_{\mathbf{g}} \psi_{\circ^{k} *^{m}}(\mathbf{z}) .
$$

Now rewrite the sum over $\mathbf{g} \in \mathcal{W}_{N}^{0}$ as a double sum over $\mathbf{h} \in \mathcal{W}^{I}$ and over $\mathbf{s} \in I$, using eqs.(67, 68 )

$$
P_{\eta(N, m)}(\mathbf{z}) \propto \sum_{\mathbf{h} \in \mathcal{W}^{I}} \sum_{\mathbf{s} \in I} \chi\left(T_{\mathbf{h}}\right) \chi\left(T_{\mathbf{s}}\right) T_{\mathbf{h}} T_{\mathbf{s}} \psi_{\mathrm{o}^{k} *^{m}}(\mathbf{z}) .
$$

For $\mathbf{s} \in I$ one has that $T_{\mathbf{s}} \psi_{0^{k_{*} m}}(\mathbf{z}) \propto \psi_{\mathrm{o}^{k_{*} m}}(\mathbf{z})$ and hence

$$
\sum_{\mathbf{s} \in I} \chi\left(T_{\mathbf{s}}\right) T_{\mathbf{s}} \psi_{\mathrm{o}^{k} * m}(\mathbf{z}) \propto \psi_{\mathrm{o}^{k_{*} m}}(\mathbf{z}) .
$$

Therefore we obtain

$$
P_{\eta(N, m)}(\mathbf{z}) \propto \sum_{\mathbf{h} \in \mathcal{W}^{I}} \chi\left(T_{\mathbf{h}}\right) T_{\mathbf{h}} \psi_{\circ^{k} *^{*}}(\mathbf{z})=\sum_{w \in \mathcal{Q}(N, m)} \psi_{w}(\mathbf{z}) .
$$

In order to fix the proportionality constant we recall that $P_{\eta(N, m)}(\mathbf{z})$ is normalized in such a way that the monomial $\mathbf{z}^{\mu(N-m, m)}$ has coefficient 1. On the other side, in the sum defining $Z_{N, m}(\mathbf{z})$, such monomial comes 
only from the component $\psi_{\circ^{k}{ }^{m}}(\mathbf{z})$ and has coefficient 1 . Therefore we conclude that the proportionality factor is 1 .

Actually we can do even better and consider the generating function for the number of particles in the system. Calling $\bullet(w)$ the number of particles present in the state $w$, what we would like to consider is the weighted partition function

$$
Z_{N}(\xi ; \mathbf{z}):=\sum_{w \in \mathcal{Q}(N, m)} \xi^{\bullet(w)} \psi_{w}(\mathbf{z}) .
$$

In order to state our result it is convenient to switch to a notation in which the dependence on the parameters $a, b, c, d$ is explicit writing $Z_{N, m}(\mathbf{z} ; a, b, c, d)$ for the normalization. Then we have

Theorem 4. The generating function for the number of particles in the system is given by

$$
Z_{N, m}\left(\xi^{2} ; \mathbf{z} ; a, b, c, d\right)=\xi^{N-m} Z_{N, m}\left(\xi \mathbf{z} ; a_{\xi}, b_{\xi}, c_{\xi}, d_{\xi}\right) .
$$

with $a_{\xi}=\xi a, b_{\xi}=\xi b, c_{\xi}=\xi^{-1} c, d_{\xi}=\xi^{-1} d$.

This theorem is just an immediate corollary of the following

Proposition 2. For any state $w \in \mathcal{Q}(N, m)$ we have

$$
\xi^{2 \bullet(w)} \psi_{w}(\mathbf{z} ; a, b, c, d)=\xi^{N-m} \psi_{w}\left(\xi \mathbf{z} ; a_{\xi}, b_{\xi}, c_{\xi}, d_{\xi}\right)
$$

Proof. For $w=\circ^{k} *^{m}$ eq. (174) is equivalent to eq.(63). Then we claim that if eq.(60) is true for some $w$ then it is true for all the states with the same number of first particles. Indeed if $\bullet(w)=\bullet\left(w^{\prime}\right)$, then $\psi_{w^{\prime}}$ is obtained from $\psi_{w}$ through the action of the sole operators $T_{i}$ with $1 \leq i \leq N-1$, which are homogeneous in the variables $\mathbf{z}$.

The last step is an induction on the number of first class particles. Assume eq. (60) is true for all $w$ such that $\bullet(w) \leq k$. Then consider a state $\bar{w}$ with $\bullet(\bar{w})=n$, of the form $\bar{w}=\tilde{w} \circ$. Writing $\psi_{\tilde{w} \circ}(\mathbf{z})$ as

$$
\psi_{\tilde{w} \circ}(\mathbf{z})=z_{N}^{-1} \phi^{(-1)}\left(\mathbf{z}_{\widehat{N}}\right)+\phi^{(0)}\left(\mathbf{z}_{\widehat{N}}\right),
$$

the relation (60) translates into

$$
\begin{aligned}
& \xi^{k} \phi^{(0)}\left(\mathbf{z}_{\widehat{N}} ; a, b, c, d\right)=\xi^{N-m} \phi^{(0)}\left(\xi \mathbf{z}_{\widehat{N}} ; a_{\xi}, b_{\xi}, c_{\xi}, d_{\xi}\right) \\
& \xi^{k} \phi^{(1)}\left(\mathbf{z}_{\widehat{N}} ; a, b, c, d\right)=\xi^{N-m-1} \phi^{(1)}\left(\xi_{\widehat{N}}, a_{\xi}, b_{\xi}, c_{\xi}, d_{\xi}\right) .
\end{aligned}
$$

Acting with $t_{N}^{-\frac{1}{2}} T_{N}^{-1}$ on the component $\psi_{\tilde{w} \circ}(\mathbf{z})$ we get

$$
\psi_{\tilde{w} \bullet}(\mathbf{z})=\left(z_{N}-c^{-1}-d^{-1}\right) \phi^{(-1)}(\hat{\mathbf{z}})-c^{-1} d^{-1} \phi^{(0)}(\hat{\mathbf{z}}) .
$$

Using this expression and eqs.(75) we easily verify that $\psi_{\tilde{w} \bullet}(\mathbf{z})$ satisfies eq. (60). 
A more explicit formula for the weighted partition function $Z_{N, m}\left(\xi^{2} ; \mathbf{z}\right)$, which is well suited for asymptotic analysis, is obtained in terms of an integral representation of the Macdonald-Koornwinder polynomials 30. (see Appendix A.2). Assuming $t<1$ we can write

$$
\begin{aligned}
& Z_{N, m}\left(\xi^{2} ; \mathbf{z}\right)=r_{m}^{-1}\left(a_{\xi}, b_{\xi}, c_{\xi}, d_{\xi} \mid t\right) \times \\
& \quad \xi^{N-m} \oint_{\mathcal{C}} \frac{d x}{4 \pi i x} \Pi(\mathbf{z}, x) w\left(x ; a_{\xi}, b_{\xi}, c_{\xi}, d_{\xi} \mid t\right) p_{m}\left(x ; a_{\xi}, b_{\xi}, c_{\xi}, d_{\xi} \mid t\right)
\end{aligned}
$$

where $p_{m}(x ; a, b, c, d \mid t)$ is the $m$-th Askey-Wilson polynomial of base $t$ in the variable $\frac{x+x^{-1}}{2}, w(x ; a, b, c, d \mid t)$ is the Askey-Wilson kernel

$$
w(x ; a, b, c, d \mid t)=\frac{\left(x^{2}, x^{-2} ; t\right)_{\infty}}{\left(a x, a x^{-1}, b x, b x^{-1}, c x, c x^{-1}, d x, d x^{-1} ; t\right)_{\infty}},
$$

while $\Pi(\mathbf{z}, \mathbf{x})=\prod_{1 \leq i \leq N}\left(z_{i}+z_{i}^{-1}-x-x^{-1}\right)$ and the normalization constant $r_{m}$ is given by

$$
r_{m}(a, b, c, d \mid t)=\frac{\left(a b c d t^{2 m} ; t\right)_{\infty}}{\left(t^{m+1}, a b t^{m}, a c t^{m}, a d t^{m}, b c t^{m}, b d t^{m}, c d t^{m} ; t\right)_{\infty}}
$$

The contour of integration $\mathcal{C}$ encircles the poles in $a_{\xi} t^{k}, b_{\xi} t^{k}, c_{\xi} t^{k}, d_{\xi} t^{k}$ $\left(k \in \mathbb{Z}_{+}\right)$and excludes all the others.

In the homogeneous limit, our result for $Z_{N, m}(\xi ; \mathbf{z})$ looks quite different and simpler than the analogous result in [39], where Uchiyama computes the grand partition function. A more direct comparison can be done for $m=0$ with the expression for $Z_{N, m}(\xi ; \mathbf{1})$ presented in [40, Eq.(6.2)] (their parameters $a, b, c, d, q$ are related to ours by $\left.q=t^{-1}, a \leftrightarrow-a^{-1}, d \leftrightarrow-d^{-1}, b \leftrightarrow-c^{-1}\right)$. Since $p_{0}(x \mid t)=1$, we see that the integral in eq.(76) coincides with the one in [40, Eq.(6.2)], nonetheless the two formulas differ by a prefactor which depends on $\xi$ (and therefore cannot be accounted by a different normalization of the wave function), but not on the size $N$ of the system. This gives rise at finite size to different predictions for the average occupation number of first class particles.

\section{RECURSION RELATIONS}

In this Section we will discuss some relations between solutions of the exchange-reflection equations for systems of different size and different values of the parameters $a, b, c, d$. This will allow us to derive some contiguous relations for Askey-Wilson polynomials. Whenever we shall compare solutions corresponding to different values of the parameter $a, b, c, d$ we shall keep explicit the dependence on these parameters. 
Theorem 5. The following three kinds of recursive relations holds

$$
\begin{gathered}
\psi_{w *}(\mathbf{z} ; a, b, c, d)=\psi_{w}\left(\mathbf{z}_{\widehat{N}} ; a, b, t c, t d\right) \\
\psi_{* w}(\mathbf{z} ; a, b, c, d)=\psi_{w}\left(\mathbf{z}_{\hat{1}} ; t a, t b, c, d\right) . \\
\psi_{w \circ}(\mathbf{z})+c d \psi_{w \bullet}(\mathbf{z})=\frac{\left(1-c z_{N}\right)\left(1-d z_{N}\right)}{z_{N}} \psi_{w}\left(\mathbf{z}_{\widehat{N}}\right) \\
a b \psi_{\circ w}(\mathbf{z})+\psi_{\bullet w}(\mathbf{z})=\frac{\left(a-z_{1}\right)\left(b-z_{1}\right)}{z_{1}} \psi_{w}\left(\mathbf{z}_{\hat{1}}\right) \\
\left.\psi_{w \circ}(\mathbf{z} ; a, b, c, d)\right|_{z_{N}=c^{-1}}=K_{R}(c) \psi_{w}\left(\mathbf{z}_{\widehat{N}} ; a, b, t c, d\right) \\
\left.\psi_{w \circ}(\mathbf{z} ; a, b, c, d)\right|_{z_{N}=d^{-1}}=K_{R}(d) \psi_{w}\left(\mathbf{z}_{\widehat{N}} ; a, b, c, t d\right) \\
\left.\psi_{w \bullet}(\mathbf{z} ; a, b, c, d)\right|_{z_{N}=c^{-1}}=-c^{-1} d^{-1} K_{R}(c) \psi_{w}\left(\mathbf{z}_{\widehat{N}} ; a, b, t c, d\right) \\
\left.\psi_{w \bullet}(\mathbf{z} ; a, b, c, d)\right|_{z_{N}=d^{-1}}=-c^{-1} d^{-1} K_{R}(d) \psi_{w}\left(\mathbf{z}_{\widehat{N}} ; a, b, c, t d\right) \\
\left.\psi_{\circ w}(a, b, c, d ; \mathbf{z})\right|_{z_{1}=a}=K_{L}(a) \psi_{w}\left(t a, b, c, d ; \mathbf{z}_{\hat{1}}\right) \\
\left.\psi_{\circ w}(a, b, c, d ; \mathbf{z})\right|_{z_{1}=b}=K_{L}(b) \psi_{w}\left(a, t b, c, d ; \mathbf{z}_{\hat{1}}\right) \\
\left.\psi_{\bullet w}(a, b, c, d ; \mathbf{z})\right|_{z_{1}=a}=-a b K_{L}(a) \psi_{w}\left(t a, b, c, d ; \mathbf{z}_{\hat{1}}\right) \\
\left.\psi_{\bullet w}(a, b, c, d ; \mathbf{z})\right|_{z_{1}=b}=-a b K_{L}(b) \psi_{w}\left(a, t b, c, d ; \mathbf{z}_{\hat{1}}\right)
\end{gathered}
$$

where $\mathbf{z}_{\hat{\ell}}$ means that the variable $z_{\ell}$ is absent and

$$
\begin{aligned}
& K_{R}(x)=-\frac{\left(1-a x t^{m}\right)\left(1-b x t^{m}\right) c d x^{-1}}{1-a b c d t^{2 m}} \\
& K_{L}(x)=\frac{\left(1-c x t^{m}\right)\left(1-d x t^{m}\right) x^{-1}}{1-a b c d t^{2 m}}
\end{aligned}
$$

Proof. First we claim that if eqs. $(79,82)$ hold for some $w \in \mathcal{Q}(N, m)$ then they hold for any configurations in $\mathcal{Q}(N, m)$. Indeed, for the equations in which the particle configuration at site $N$ is fixed, one can modify $w$ by acting on both sides of the equalities either with operators $T_{i}$ with $0 \leq i<N-1$, while for the equations in which it is the first site configuration to be fixed, one can modify $w$ by acting with operators $T_{i}$ with $1<i \leq N$.

We shall use the previous remark to prove eqs. (79,80). We could use it also to prove eqs. (81,82) but we prefer to adopt a different strategy.

The first equation of (79), in the case $w=\circ^{k} *^{m}$ coincides with eq. (61), while the second equation of (79) for $w=*^{m} \circ^{k}$ coincides with eq. (64). 
In order to prove the second of eqs. (80) for $w=\circ^{k-1} *^{m}$, we use the integral expression of $\psi_{\mathrm{o}^{k} m}(a, b, c, d ; \mathbf{z})$ given by eq.(55)

$$
\begin{gathered}
a b \psi_{\circ \circ^{k-1} *^{m}}(\mathbf{z})+\psi_{\bullet \circ^{k-1} *^{m}}(\mathbf{z})= \\
\left(a b+t_{0}^{\frac{1}{2}} T_{0}\right) \psi_{\circ_{*}{ }^{m}}(\mathbf{z})= \\
\left(a b+t_{0}^{\frac{1}{2}} T_{0}\right) \oint_{0} \frac{d w}{2 \pi i w} F_{m}(w) \prod_{j=1}^{k}\left(w^{-1}+z_{j}^{-1}\right)= \\
\frac{\left(a-z_{1}\right)\left(b-z_{1}\right)}{z_{1}} \oint_{0} \frac{d w}{2 \pi i w} F_{m}(w) \prod_{j=2}^{k}\left(w^{-1}+z_{j}^{-1}\right)= \\
\frac{\left(a-z_{1}\right)\left(b-z_{1}\right)}{z_{1}} \psi_{\circ^{k-1} *^{m}}\left(\mathbf{z}_{\hat{1}}\right)
\end{gathered}
$$

where in the third equality we have used the identity

$$
\left(a b+t_{0}^{\frac{1}{2}} T_{0}\right)\left(w^{-1}+z_{1}^{-1}\right)=\frac{\left(a-z_{1}\right)\left(b-z_{1}\right)}{z_{1}} .
$$

In a similar way one proves the first of eqs. 80 .

For the proof of eqs. (81) we adopt a different strategy: we show that the 1.h.s. satisfies the same exchange-reflection equations as the r.h.s. and therefore by Theorem 2 they must be proportional.

Lets look at the first of these equations, since we are comparing systems of different length we must distinguish between operators representing the Affine Hecke algebra of different rank and different parameters therefore just for the present proof we shall use an heavier notations. For the case of rank $N$ with parameter $c$ highlighted we shall write the generators as $T_{i}^{(N)}(c)$. In terms of divided difference operators we have of course $T_{i}^{(N)}(c)=T_{i}^{(N-1)}\left(c^{\prime}\right)$ for $i<N-2$ and any $c, c^{\prime}$, therefore both sides of the first of eqs. (81) satisfies eqs. (50) f51) for $i<N-2$ and $T_{i}^{(N)}(t c)$. It remains to prove that the r.h.s. satisfies eqs.(51) for $i=N-1$. If $w=\tilde{w} *$ then $\psi_{w \circ}$ does not depend on $z_{N-1}$ so in particular it is invariant under $z_{N-1} \leftrightarrow z_{N-1}^{-1}$. If $w=\tilde{w} \circ$ then $\psi_{\tilde{w} \circ \circ}$ is symmetric in $z_{N-1}, z_{N}$ and we have

$$
\psi_{\tilde{w} \bullet \circ}(\mathbf{z})=\left((-t c d)^{\frac{1}{2}} T_{N}^{(N)}(c) T_{N-1}^{(N)}(c)\right)^{-1} \psi_{\tilde{w} \circ \circ}(\mathbf{z})
$$

It is straightforward to verify that for any symmetric function $H(x, y)$ one has the following identity

$$
\left.\left(T_{N}^{(N)}(c) T_{N-1}^{(N)}(c)\right)^{-1} H\left(z_{N-1}, z_{N}\right)\right|_{z_{N}=c^{-1}}=\left(T_{N-1}^{(N-1)}(t c)\right)^{-1} H\left(z_{N-1}, c^{-1}\right) .
$$


This means that after specialization $z_{N}=c^{-1}$ in eq.(84) we get

$$
\left.\psi_{\tilde{w} \bullet \circ}(\mathbf{z})\right|_{z_{N}=c^{-1}}=\left.\left(T_{N-1}^{(N-1)}(t c)\right)^{-1} \psi_{\tilde{w} \circ \circ}(\mathbf{z})\right|_{z_{N}=c^{-1}}
$$

It remains to fix the proportionality constant $K_{R}(c)$. This is given by the coefficient of the monomial $\prod_{j=1}^{k} z_{j}^{-1}$ in $\left.\psi_{*^{m} \circ^{k}}(\mathbf{z} ; a, b, c, d)\right|_{z_{N}=c^{-1}}$, since the same monomial has coefficient 1 in $\psi_{*^{m}{ }^{k-1}}\left(\mathbf{z}_{\widehat{N}} ; a, b, t c, d\right)$. We get

$$
K_{R}(c)=c+t^{m} h_{1}\left(a, b, t^{m} c, t^{m} d \mid t\right)=\frac{\left(-1+a c t^{m}\right)\left(-1+b c t^{m}\right) d}{-1+a b c d t^{2 m}} .
$$

In a similar way one proves the identities for the specializations $z_{1}=$ $a, b$.

Since $\psi_{w o}(\mathbf{z} ; a, b, c, d)$ and $\psi_{w \bullet}(\mathbf{z} ; a, b, c, d)$ are polynomials of degree 1 in $z_{N}$ and $z_{N}^{-1}$ respectively, they are recursively determined by the two equations (81). At the level of partition function the recursions (79,81) imply

$$
\begin{aligned}
& \left.Z_{N, m}(\mathbf{z} ; a, b, c, d)\right|_{z_{N}=c^{-1}}=Z_{N-1, m-1}\left(\mathbf{z}_{\widehat{N}} ; a, b, t c, t d\right)+ \\
& \frac{\left(1-a c t^{m}\right)\left(1-b c t^{m}\right)(1-d c)}{c\left(1-a b c d t^{2 m}\right)} Z_{N-1, m-1}\left(\mathbf{z}_{\widehat{N}} a, b, t c, d\right) .
\end{aligned}
$$

By using the integral formula (71) in the previous equation, we derive a contiguous relation for Askey-Wilson polynomials which is not difficult to prove by direct means 2

$$
\begin{aligned}
& \text { (86) }\left(1-c d t^{m}\right) P_{m}(x ; a, b, c, d \mid t)-(1-c d) P_{m}(x ; a, b, t c, d \mid t) \\
& -c d\left(1-t^{m}\right)\left(1-a b t^{m-1}\right)\left(z+z^{-1}-d-d^{-1}\right) P_{m-1}(x ; a, b, t c, t d \mid t)=0 .
\end{aligned}
$$

On the other hand eq. (85) and its analog for $z_{N}=d^{-1}$, determine $Z_{N, m}(\mathbf{z} ; a, b, c, d)$ uniquely. Therefore assuming eq.(86), we could obtain a proof of the integral formula (71), without employing Mimachi's formula.

Another consequence of the recursions (81,82) concerns the coefficients $h_{n}$ in the expansion of $\psi_{\mathrm{o}^{*} *^{m}}$. Indeed by applying eqs. (81,82) to the case $w=0^{n}$ we get

$$
\begin{aligned}
h_{n+1}(a, b, c, d)+c h_{n}(a, b, c, d) & =-\frac{(1-a c)(1-b c) d}{(1-a b c d)}, h_{n}(a, b, t c, d) \\
h_{n+1}(a, b, c, d)+a^{-1} h_{n}(a, b, c, d) & =\frac{(1-a c)(1-a d) a^{-1}}{1-a b c d} h_{n}(t a, b, c, d) .
\end{aligned}
$$

\footnotetext{
${ }^{2}$ Several contiguous/difference relations for Askey-Wilson polynomials emerging from our discussion are presented in Appendix A.3
} 
Actually these two equations are equivalent thanks to the duality relation (62). The previous two equations can be written as contiguous or difference equations for Al-Salam Chihara polynomials

$$
\begin{aligned}
& a Q_{n+1}(z ; a, b \mid t)-\left(1-a b t^{n}\right) Q_{n}(z ; a, b \mid t) \\
& -a\left(z+z^{-1}-a-a^{-1}\right) Q_{n}(z ; t a, b \mid t)=0 \\
& \begin{aligned}
Q_{n+1}(z ; a, b \mid t)-z\left(1-a b t^{n}\right) Q_{n}(z ; a, b \mid t) \\
-t^{n / 2} \frac{(1-a z)(1-b z)}{z} Q_{n}\left(t^{\frac{1}{2}} z ; t^{\frac{1}{2}} a, t^{\frac{1}{2}} b \mid t\right)=0 .
\end{aligned}
\end{aligned}
$$

Both equations can be lifted to contiguous or difference relations for Askey-Wilson polynomials (see Appendix A.3).

\section{DENSITY AND CURRENT}

In order to compute physical quantities, like average density of first class particles or current, we need to specialize the spectral parameters $\mathbf{z}=\mathbf{1}$. While for the density this is straightforward

$$
\left\langle\rho_{N, m}^{\bullet}\right\rangle=\left.\left(\frac{1}{N} \frac{\partial}{\partial \xi} \log Z_{N, m}(\xi ; \mathbf{1})\right)\right|_{\xi=0},
$$

for the steady current $\left\langle J_{N, m}\right\rangle$ we need to make an intermediate step and define an inhomogeneous version of that quantity

$$
J_{N, m}(\mathbf{z}):=\frac{1}{Z_{N, m}(\mathbf{z})} \sum_{w \in \mathcal{Q}(N, m)} \alpha \psi_{\text {ow }}(\mathbf{z})-\gamma \psi_{\bullet w}(\mathbf{z}),
$$

which under specialization $\mathbf{z}=\mathbf{1}$ reduces to the homogeneous case,

$$
\left\langle J_{N, m}\right\rangle=J_{N, m}(\mathbf{1}) .
$$

Using the fact that $\alpha / \gamma=-a b$ and the first of eqs.(80), we get an explicit formula for $J_{N, m}(\mathbf{z})$

$$
J_{N, m}(\mathbf{z})=-\gamma \frac{\left(a-z_{1}\right)\left(b-z_{1}\right)}{z_{1}} \frac{Z_{N-1, m}\left(\mathbf{z} \backslash z_{1}\right)}{Z_{N, m}(\mathbf{z})} .
$$

After specialization $\mathbf{z}=\mathbf{1}$, we obtain

$$
\left\langle J_{N, m}\right\rangle=\left(t^{\frac{1}{2}}-t^{-\frac{1}{2}}\right) \frac{Z_{N-1, m}(\mathbf{1})}{Z_{N, m}(\mathbf{1})} .
$$


5.1. Thermodynamic limit. Since the stationary current and the average first particle density have been expressed in terms of the weighted partition function, in order to determine the behavior of such quantities in the thermodynamic limit we need to work out the asymptotic behavior of $Z_{N, m}\left(\xi^{2} ; \mathbf{1}\right)$ when $N$ and $m$ tend to infinity at fixed density of second class particles $\rho_{*}=m / N$.

If we assume $t<1$, we can use eq.(76). In that equation the prefactor $r_{m}^{-1}\left(a_{\xi}, b_{\xi}, c_{\xi}, d_{\xi} \mid t\right)$ for $m \rightarrow \infty$ goes to a constant $\kappa$, therefore we just need to consider the integral, which in the homogeneous specialization reads

$$
\oint_{\mathcal{C}} \frac{d x}{4 \pi i x} \frac{\left(x^{2}, x^{-2} ; t\right)_{\infty}\left(\xi+\xi^{-1}-x-x^{-1}\right)^{N} p_{m}\left(x ; a_{\xi}, b_{\xi}, c_{\xi}, d_{\xi} \mid t\right)}{\left(a_{\xi} x, a_{\xi} x^{-1}, b_{\xi} x, b_{\xi} x^{-1}, c_{\xi} x, c_{\xi} x^{-1}, d_{\xi} x, d_{\xi} x^{-1} ; t\right)_{\infty}}
$$

For the large $N, m$ we can use the asymptotic formula eq.(112) for Askey-Wilson polynomials and we arrive at

$$
Z_{N, m}\left(\xi^{2} ; \mathbf{1}\right) \simeq k \xi^{N-m} \oint_{\mathcal{C}} \frac{d x}{4 \pi i x} \frac{\left(x^{-2} ; t\right)_{\infty}\left(\xi+\xi^{-1}-x-x^{-1}\right)^{N} x^{-m}}{\left(a_{\xi} x^{-1}, b_{\xi} x^{-1}, c_{\xi} x^{-1}, d_{\xi} x^{-1} ; t\right)_{\infty}}
$$

For $\xi \sim 1$ the function

$$
f(x)=\log \left(\xi+\xi^{-1}-x-x^{-1}\right)-\rho_{*} \log (x)
$$

has a saddle point at

$$
x_{\xi}=x_{0}+O((\xi-1)), \quad x_{0}=\frac{1+\rho_{*}}{-1+\rho_{*}} \leq-1 .
$$

Now recall that for $0<t<1$ the parameters $a, b, c, d$ span the range $a, c<0$ and $0<b, d<1$. When $x_{0}<a, c<0$ we can deform the contour of integration to the steepest descent path that passes through $x\left(\rho_{*} ; \xi\right)$ and we easily find

$$
Z_{N, m}\left(\xi^{2} ; \mathbf{1}\right) \simeq \frac{k \xi^{N-m}}{\sqrt{8 \pi f^{\prime \prime}\left(x_{\xi}\right) N}} \frac{\left(x_{\xi}^{-2} ; t\right)_{\infty}\left(\xi+\xi^{-1}-x_{\xi}-x_{\xi}^{-1}\right)^{N} x_{\xi}^{-m}}{\left(a_{\xi} x_{\xi}^{-1}, b_{\xi} x_{\xi}^{-1}, c_{\xi} x_{\xi}^{-1}, d_{\xi} x_{\xi}^{-1} ; t\right)_{\infty}} .
$$

When at least one among $a$ and $c$ is less then $x_{0}$ then the dominating contribution comes from the pole around $a_{\xi}$ if $a<c$ or around $c_{\xi}$ if $c<a$, and we get

$$
Z_{N, m}\left(\xi^{2} ; \mathbf{1}\right) \simeq \begin{cases}\frac{k \xi^{N-m}\left(a_{\xi}^{-2} ; t\right)_{\infty}\left(\xi+\xi^{-1}-a_{\xi}-a_{\xi}^{-1}\right)^{N} a_{\xi}^{-m}}{2 a_{\xi}\left(t, b_{\xi} a_{\xi}^{-1}, c_{\xi} a_{\xi}^{-1}, d_{\xi} a_{\xi}^{-1} ; t\right)_{\infty}} & a<c, x_{0} \\ \frac{k \xi^{N-m}\left(c_{\xi}^{-2} ; t\right)_{\infty}\left(\xi+\xi^{-1}-c_{\xi}-c_{\xi}^{-1}\right)^{N} c_{\xi}^{-m}}{2 c_{\xi}\left(t, b_{\xi} c_{\xi}^{-1}, a_{\xi} c_{\xi}^{-1}, d_{\xi} c_{\xi}^{-1} ; t\right)_{\infty}} & c<a, x_{0}\end{cases}
$$


Let us now call $J_{\rho_{*}}$ and $\rho_{\rho_{*}}^{\bullet}$ the steady state current and density of first class particle in the thermodynamic limit. Combining eqs. (89, [92) with eqs.967) we obtain

$$
\begin{aligned}
& J_{\rho_{*}}=\frac{\left(t^{\frac{1}{2}}-t^{-\frac{1}{2}}\right)\left(1-\rho_{*}^{2}\right)}{4}, \quad \rho_{\rho_{*}}^{\bullet}=\frac{1-\rho_{*}}{2}, \quad \text { for } \quad x_{0}<a, c \\
& J_{\rho_{*}}=\frac{a\left(t^{-\frac{1}{2}}-t^{\frac{1}{2}}\right)}{(1-a)^{2}}, \quad \rho_{\rho_{*}}^{\bullet}=\frac{a}{a-1}-\rho_{*}, \quad \text { for } \quad a<x_{0}, c \\
& J_{\rho_{*}}=\frac{c\left(t^{-\frac{1}{2}}-t^{\frac{1}{2}}\right)}{(1-c)^{2}}, \quad \rho_{\rho_{*}}^{\bullet}=\frac{1}{1-c}, \quad \text { for } \quad c<x_{0}, a
\end{aligned}
$$

Upon redefinition $q=t^{-1}, a \leftrightarrow-a^{-1}, d \leftrightarrow-d^{-1}, b \leftrightarrow-c^{-1}$ our results are in agreement with 39 .

\section{Conclusion}

In this paper we have analyzed the steady state of the Asymmetric Simple Exclusion process with open boundaries and second class particles by deforming it through the introduction of spectral parameters, in a way which is dictated by the integrable structure of the model. The (unnormalized) probabilities of the particle configurations get promoted to Laurent polynomials in the spectral parameters, that are constructed in terms of non-symmetric Koornwinder polynomials. Moreover we have shown that the partition function coincides with a symmetric Macdonald-Koornwinder polynomial. As an outcome we have computed the steady current and the average density of first class particles. It is interesting to pursue further the analysis of the inhomogeneous model and compute other quantities like correlation functions.

In a recent preprint [13] Corteel and Williams have uncovered a different connection between open 2ASEP with open boundaries a second class particles and the theory of Koornwinder polynomials. It would be extremely interesting to investigate the relation of their findings with the algebraic structure presented in our paper.

It is clear that our approach can be applied to other stochastic interacting particle systems as soon as one recognizes that the Markov matrix is integrable. The most straightforward generalization of the present paper would consist in a system with more than two species of particles and exchange rates that arise from a higher rank quotient of the affine Hecke algebra of type $\tilde{\mathcal{C}}_{N}$. A more interesting class of systems that can be treated with our approach [4], arise from the classification of the stochastic boundary scattering matrices of Crampe et al. [15]. One could also consider systems with periodic boundary conditions. 
While for just a single class of particles the steady state is simply uniform, this is no longer true if one allows for more classes of particles, and the nontrivial steady state can be analyzed using the approach presented here [7, 6].

\section{Appendix A. KoORnWinder, MaCdonald-KoORnWinder And ASKEY-WILSON POLYNOMIALS}

In this Appendix we shall the definitions of Koornwinder, MacdonaldKoornwinder and Askey-Wilson polynomials, and a few of their properties we needed in the paper.

A.1. Nonsymmetric Koornwinder polynomials. In order to introduce the Non-symmetric Koornwinder polynomials one considers the action, in the Noumi representation, of the commutative sub-algebra $\mathcal{Y}_{N}$ on $\mathbb{C}\left[z_{1}^{ \pm 1}, \ldots, z_{N}^{ \pm 1}\right]$. It turns out that such action is triangular with respect to the order $\preceq$ on the monomials [37]

$$
Y_{i} \mathbf{z}^{\alpha}=\omega_{i}(\alpha) \mathbf{z}^{\alpha}+\sum_{\mathbf{z}^{\beta} \prec \mathbf{z}^{\alpha}} \tilde{c}_{\beta} \mathbf{z}^{\beta} .
$$

in order to write the eigenvalues $\omega_{i}(\alpha)$ we need to introduce $w_{\alpha}$, which is the minimal length element in $\mathcal{W}_{N}^{0}$ such that $w_{\alpha} \alpha^{+}=\alpha$ and $\rho=$ $\{1,2, \ldots, N\} \in \mathbb{Z}$. Then we can write

$$
\omega_{i}(\alpha)=q^{\alpha_{i}}\left(\sqrt{q^{-1} a b c d} t^{N-\left|\left(w_{\alpha} \rho\right)_{i}\right|}\right)^{\operatorname{sign}\left(\left(w_{\alpha} \rho\right)_{i}\right)}
$$

The common eigenfunctions of $\mathcal{Y}_{N}$ are the non-symmetric Koornwinder polynomials $E_{\alpha}(\mathbf{z})$ 32]

$$
\begin{aligned}
& E_{\alpha}(\mathbf{z})=\mathbf{z}^{\alpha}+\sum_{\mathbf{z}^{\beta} \prec \mathbf{z}^{\alpha}} c_{\beta} \mathbf{z}^{\beta}, \\
& Y_{i} E_{\alpha}(\mathbf{z})=\omega_{i}(\alpha) E_{\alpha}(\mathbf{z}) \text {. }
\end{aligned}
$$

A.2. Macdonald-Koornwinder polynomials. For a partition $\lambda \in$ $\mathbb{Z}^{N}$, call $H_{\lambda}$ the subspace of $\mathbb{C}\left[z_{1}^{ \pm}, \ldots, z_{N}^{ \pm}\right]$generated by the polynomials $E_{\alpha}(\mathbf{z})$ such that $\alpha^{+}=\lambda$ is preserved by the action of $\mathcal{H}_{N}$ and for generic values of the parameters $a, b, c, d, q, t$ it forms an irreducible representation. The subspace $H_{\lambda}^{\mathcal{W}_{N}^{0}}$, invariant under the action of $\mathcal{W}_{N}^{0}$ is one-dimensional and is generated by $P_{\lambda}(\mathbf{z})$, the symmetric MacdonaldKoornwinder polynomial in $N$ variables $\mathbf{z}$, associated to the partition $\lambda$ [26, 32]. Before providing a definition and discussion of MacdonaldKoornwinder polynomials let us state one of the main results we need. 
Theorem 6 ([32, Corollary 6.6). Let $\chi: \mathcal{H}_{N} \rightarrow \mathbb{C}$ be the one dimensional representation of $\mathcal{H}_{N}$ defined on the generators by

$$
\chi\left(T_{i}\right)=t_{i}^{\frac{1}{2}} \text {. }
$$

Then we have

$$
P_{\alpha^{+}}(\mathbf{z}) \propto \sum_{w \in W_{N}^{0}} \chi\left(T_{w}\right) T_{w} E_{\alpha}(\mathbf{z})
$$

For a fixed $N$, the Macdonald-Koornwinder polynomials are Laurent polynomials in $N$ variables, invariant under $\mathcal{W}_{N}^{0}$, which are eigenvectors of the following q-difference operator

$$
\mathcal{D}_{q, t}=\sum_{i=1}^{N} \Phi_{i}\left(z_{i}\right)\left(T_{q, z_{i}}-1\right)+\Phi_{i}\left(z_{i}^{-1}\right)\left(T_{q, z_{i}}^{-1}-1\right)
$$

where $T_{q, z_{i}}$ is the $i$-th q-shift operator

$$
T_{q, z_{i}} f\left(z_{1}, \ldots, z_{i}, \ldots, z_{N}\right)=f\left(z_{1}, \ldots, q z_{i}, \ldots, z_{N}\right)
$$

and

$$
\Phi_{i}(z)=\frac{(1-a z)(1-b z)(1-c z)(1-d z)}{\left(1-z^{2}\right)\left(1-q z^{2}\right)} \prod_{\substack{j=1 \\ j \neq i}}^{N} \frac{\left(1-t z z_{j}\right)\left(1-t z z_{j}^{-1}\right)}{\left(1-z z_{j}\right)\left(1-z z_{j}^{-1}\right)}
$$

The Macdonald-Koornwinder polynomials in $N$ variables, $P_{\lambda}(\mathbf{z})$, are labeled by partitions $N$ parts, which parametrize the eigenvalue of $\mathcal{D}_{q, t}$, i.e. the polynomials $P_{\lambda}(\mathbf{z})$ are characterized by the equations

$$
\mathcal{D}_{q, t} P_{\lambda}(\mathbf{z})=d_{\lambda} P_{\lambda}(\mathbf{z})
$$

with

$$
d_{\lambda}=\sum_{i=1}^{N}\left[q^{-1} a b c d t^{2 n-i-1}\left(q^{\lambda_{i}}-1\right)+t^{i-1}\left(q^{-\lambda_{i}}-1\right)\right]
$$

together with the condition that the coefficient of $\mathbf{z}^{\lambda}$ in $P_{\lambda}(\mathbf{z})$ is 1 . Of course the Macdonald-Koornwinder polynomials depend on the parameter $a, b, c, d, t, q$ but we shall write this dependence explicitly (writing $\left.P_{\lambda}(\mathbf{z})=P_{\lambda}(\mathbf{z} ; a, b, c, d ; q ; t)\right)$ only when needed.

An important property of the Macdonald-Koornwinder polynomials (which could be used actually to give an alternative definition) is their orthogonality with respect to a certain scalar product. Let assume for convenience $|q|<1$ and recall the usual notation for the q-Pochhammer symbol

$$
(a ; q)_{n}=\prod_{i=0}^{n-1}\left(1-a q^{i}\right) \quad\left(a_{1}, a_{2} \ldots, a_{j} ; q\right)_{n}=\prod_{i=1}^{j}\left(a_{i} ; q\right)_{n} .
$$


Let us define the kernel

$$
\Delta_{N}(t, q ; \mathbf{z})=\prod_{\substack{1 \leq i<j \leq N \\ \epsilon_{1}, \epsilon_{2}= \pm 1}} \frac{\left(z_{i}^{\epsilon_{1}} z_{j}^{\epsilon_{2}} ; q\right)_{\infty}}{\left(t z_{i}^{\epsilon_{1}} z_{j}^{\epsilon_{2}} ; q\right)_{\infty}} \prod_{i=1}^{N} \frac{\left(z_{i}^{2 \epsilon} ; q\right)_{\infty}}{\left(a z_{i}^{\epsilon}, b z_{i}^{\epsilon}, c z_{i}^{\epsilon}, d z_{i}^{\epsilon} ; q\right)_{\infty}}
$$

and the scalar product on $\mathbb{C}\left[z_{1}^{ \pm}, \ldots, z_{N}^{ \pm}\right]$

$$
\langle p, q\rangle:=\oint_{\mathcal{C}} \prod_{j=1}^{N} \frac{d z_{j}}{4 \pi i z_{j}} \Delta_{N}(t, q ; \mathbf{z}) p(\mathbf{z}) q\left(\mathbf{z}^{-1}\right)
$$

where the contour of integration $\mathcal{C}$ encircles the poles in $a q^{k}, b q^{k}, c q^{k}, d q^{k}$ $\left(k \in \mathbb{Z}_{+}\right)$and excludes all the others. The Macdonald-Koornwinder polynomials corresponding to different partitions are orthogonal with respect to such scalar product

$$
\left\langle P_{\lambda}, P_{\mu}\right\rangle=0 \quad \text { if } \quad \lambda \neq \mu .
$$

When the partition $\lambda=\{m\}$, i.e. when $\lambda$ consists of just a single part of length $m$, the Macdonald-Koornwinder polynomials are polynomials in one variable $z$ independent of the variable $t$. They correspond (up to a factor and change of variables $\left.x=\left(z+z^{-1}\right) / 2\right)$ to the Askey-Wilson polynomials $\left.p_{m}(z ; a, b, c, d \mid q)\right)^{3}[2,22$ ]

$$
P_{\{m\}}(\mathbf{z}) \propto p_{m}(z ; a, b, c, d \mid q) .
$$

A.3. Askey-Wilson polynomials. Let us recall the definition of the Askey-Wilson polynomials in terms of a terminating hypergeometric function

$$
p_{n}(z ; a, b, c, d \mid q)=\frac{(a b, a c, a d ; q)_{n}}{a^{n}}{ }_{4} \phi_{3}\left(\begin{array}{c}
q^{-n}, q^{n-1} a b c d, a z, a z^{-1} \\
a b, a c, a d
\end{array} ; q, q\right)
$$

where the basic hypergeometric functions ${ }_{r} \phi_{s}$ are defined by

$$
{ }_{r} \phi_{s}\left(\begin{array}{c}
a_{1}, a_{2}, \ldots, a_{s} \\
b_{1}, \ldots, b_{r}
\end{array} ; q, z\right)=\sum_{n=0}^{\infty} \frac{\left(a_{1}, \ldots, a_{r} ; q\right)_{n}}{\left(q, b_{1}, \ldots, b_{s} ; q\right)_{n}}\left[(-1)^{n} t^{\left(\begin{array}{c}
n \\
2
\end{array}\right)}\right]^{s+1-r} z^{n}
$$

As for the case of Macdonald-Koornwinder polynomials, whenever the parameters $a, b, c, d$ are clear from the context we shall use a lighter notation writing $p_{n}(z \mid q)=p_{n}(z ; a, b, c, d \mid q)$. The Askey-Wilson polynomials satisfy orthogonality relations that correspond to the case $N=1$ of the orthogonality relation for Koornwinder-Macdonald polynomials.

\footnotetext{
${ }^{3}$ In the literature the Askey-Wilson polynomials are usually considered as function of $x=\frac{z+z^{-1}}{2}$.
} 
Indeed for $N=1$ the kernel $\Delta_{1}(t, q ; \mathbf{z})$ becomes $t$ independent and coincides with the Askey-Wilson kernel

$$
w(z ; a, b, c, d \mid q)=\frac{\left(z^{2}, z^{-2} ; q\right)_{\infty}}{\left(a z, a z^{-1}, b z, b z^{-1}, c z, c z^{-1}, d z, d z^{-1} ; q\right)_{\infty}}
$$

and one has

$$
\oint_{\mathcal{C}} \frac{d z}{4 \pi i z} w(z \mid q) p_{n}(z \mid q) p_{m}(z \mid q)=h_{n} \delta_{n, m}
$$

where

$$
h_{n}=\frac{\left(q^{n-1} a b c d ; q\right)_{\infty}}{\left(1-q^{2 n-1} a b c d\right)\left(q^{n+1}, q^{n} a b, q^{n} a c, q^{n} a d, q^{n} b c, q^{n} b d, q^{n} c d ; q\right)_{\infty}} .
$$

An important information we need in the paper is the asymptotic form of $p_{m}(z \mid q)$ for large values of the label $m$ [22, Eq.7.5.13]

$$
p_{m}(z \mid q) \sim z^{m} B\left(z^{-1}\right)+z^{-m} B(z)
$$

with

$$
B(z)=\frac{(a z, b z, c z, d z ; q)_{\infty}}{\left(z^{2} ; q\right)_{\infty}}
$$

The specialization $c=d=0$ of the Askey-Wilson polynomials gives the so called Al-Salam Chihara polynomials [1, 25]

$$
Q_{n}(z ; a, b \mid t)=p_{n}(z ; a, b, 0,0 \mid t)
$$

The Al-Salam Chihara polynomials satisfy the following three terms recursion for

$$
Q_{n+1}(z)+\left((a+b) t^{n}-2 z\right) Q_{n}(z)+\left(1-t^{n}\right)\left(1-a b t^{n-1}\right) Q_{n-1}(z)=0 .
$$

The recursion relations presented in Section 4 motivate the presentation of several contiguous/difference relations for Askey-Wilson polynomials, which are quite straightforward to prove. They are best stated using divided difference operators

$$
\partial_{x, y} F(x, y):=\frac{F(x, y)-F(y, x)}{(x-y)}, \quad \partial_{x} F(x):=\frac{F(x)-F\left(x^{-1}\right)}{\left(x-x^{-1}\right)} .
$$


and using the variable $z$ instead of $x=\frac{z+z^{-1}}{2}$

$$
\begin{aligned}
P_{m}(z ; a, b, c, d) & =\partial_{c, d}\left(\omega^{(m, m)}(z ; a, b, c, d) P_{m}(z ; a, b, q c, d)\right) \\
P_{m-1}(z ; a, b, q c, q d) & =\partial_{c, d}\left(\omega^{(m-1, m)}(z ; a, b, c, d) P_{m}(z ; a, b, q c, d)\right) \\
P_{m+1}(z ; a, b, c, d) & =\partial_{c, d}\left(\omega^{(m+1, m)}(z ; a, b, c, d) P_{m}(z ; a, b, q c, d)\right) \\
P_{m}(z ; a, b, c, q d) & =\partial_{z}\left(\theta^{(m, m)}(z ; a, b, c, d) P_{m}\left(q^{\frac{1}{2}} z ; q^{\frac{1}{2}} a, q^{\frac{1}{2}} b, q^{\frac{1}{2}} c, q^{\frac{1}{2}} d\right)\right) \\
P_{m+1}(z ; a, b, c, d) & =\partial_{z}\left(\theta^{(m+1, m)}(z ; a, b, c, d) P_{m}\left(q^{\frac{1}{2}} z ; q^{\frac{1}{2}} a, q^{\frac{1}{2}} b, q^{\frac{1}{2}} c, q^{\frac{1}{2}} d\right)\right)
\end{aligned}
$$

with

$$
\begin{aligned}
\omega^{(m, m)}(z ; a, b, c, d) & =\frac{c d(z-c)(z c-1)}{\left(1-c d q^{m}\right) z c} \\
\omega^{(m-1, m)}(z ; a, b, c, d) & =\frac{1}{\left(1-q^{m}\right)\left(1-a b q^{m-1}\right)} \\
\omega^{(m+1, m)}(z ; a, b, c, d) & =\frac{\left(1-a d q^{m}\right)\left(1-b d q^{m}\right)(z-c)(z c-1)}{z} \\
\theta^{(m, m)}(z ; a, b, c, d) & =\frac{\left(a b c q^{m}-z\right)(1-a z)(1-b z)(1-c z)}{q^{\frac{m}{2}}\left(1-a b q^{m}\right)\left(1-a c q^{m}\right)\left(1-b c q^{m}\right) z^{2}} \\
\theta^{(m+1, m)}(z ; a, b, c, d) & =-\frac{(1-a z)(1-b z)(1-c z)(1-d z)}{q^{\frac{m}{2}} z^{2}} .
\end{aligned}
$$

\section{A.4. Cauchy formula for Macdonald-Koornwinder polynomi-}

als. We conclude this Appendix with the Cauchy formula for MacdonaldKoornwinder polynomials, due to Mimachi [30]. Let

$$
\Pi(\mathbf{z}, \mathbf{x})=\prod_{\substack{1 \leq i \leq N \\ 1 \leq j \leq M}}\left(z_{i}+z_{i}^{-1}-x_{j}-x_{j}^{-1}\right)
$$

and for a partition $\lambda \subseteq M^{N}$, i.e. a partition made of $N$ parts such that $\lambda_{i} \leq M$, define $\bar{\lambda}$, a partition of $M$ parts given by

$$
\bar{\lambda}_{j}=\#\left\{i \mid \lambda_{i}<N-i\right\} .
$$

Theorem 7 ([30], Theorem 2.1). The Macdonald-Koornwinder polynomials $P_{\lambda}(z)$ satisfy the equality

$$
\Pi(\mathbf{x}, \mathbf{z})=\sum_{\lambda \subseteq M^{N}}(-1)^{|\bar{\lambda}|} P_{\lambda}(\mathbf{z} ; q ; t) P_{\bar{\lambda}}(\mathbf{x} ; t ; q) .
$$

In the present paper we shall need just the case the case $M=1$, which combined with the orthogonality property, allows to provide an 
integral formula for the Macdonald-Koornwinder polynomials corresponding to a partition with a single column

$$
\eta(N, m)=\{\underbrace{1, \ldots, 1}_{N-m}, \underbrace{0, \ldots, 0}_{m}\}
$$

involving the Askey-Wilson polynomials of basis $t$

$$
P_{\eta(N, m)}(\mathbf{z} ; q ; t)=\left\langle\left(x+x^{-1}\right)^{m}, p_{m}\right\rangle^{-1} \oint_{\mathcal{C}} \frac{d x}{4 \pi i x} \Pi(\mathbf{z}, x) w(x \mid t) p_{m}(x \mid t)
$$

where

(118)

$$
\left\langle\left(x+x^{-1}\right)^{m}, p_{m}\right\rangle=\frac{\left(a b c d q^{2 m} ; q\right)_{\infty}}{\left(q^{m+1}, a b q^{m}, a c q^{m}, a d q^{m}, b c q^{m}, b d q^{m}, c d q^{m} ; q\right)_{\infty}}
$$

\section{REFERENCES}

1. W. A. Al-Salam and T. S. Chihara, Convolutions of orthonormal polynomials, SIAM Journal on Mathematical Analysis 7 (1976), no. 1, 16-28.

2. R. Askey and J. A. Wilson, Some basic hypergeometric orthogonal polynomials that generalize jacobi polynomials, vol. 319, American Mathematical Soc., 1985.

3. R. A Blythe and M. R. Evans, Nonequilibrium steady states of matrix-product form: a solver's guide, Journal of Physics A: Mathematical and Theoretical 40 (2007), no. 46, R333.

4. L. Cantini, Work in progress.

5. _ , qkz equations and ground state of the o (1) loop model with open boundary conditions, arXiv preprint arXiv:0903.5050 (2009).

6. __ Inhomogenous multi-species tasep on a ring with spectral parameters, (To appear).

7. L. Cantini, J. de Gier, and M. Wheeler, Matrix product formula for macdonald polynomials, arXiv preprint arXiv:1505.00287 (2015).

8. I. V. Cherednik, Factorizing particles on a half-line and root systems, Theoretical and Mathematical Physics 61 (1984), no. 1, 977-983.

9. T. Chou, K. Mallick, and R. K. P. Zia, Non-equilibrium statistical mechanics: from a paradigmatic model to biological transport, Reports on progress in physics 74 (2011), no. 11, 116601.

10. S. Corteel, R. Stanley, D. Stanton, and L. K. Williams, Formulae for askeywilson moments and enumeration of staircase tableaux, Transactions of the American Mathematical Society 364 (2012), no. 11, 6009-6037.

11. S. Corteel and L. K. Williams, Tableaux combinatorics for the asymmetric exclusion process, Advances in applied mathematics 39 (2007), no. 3, 293-310.

12. — Tableaux combinatorics for the asymmetric exclusion process and askey-wilson polynomials, Duke Mathematical Journal 159 (2011), no. 3, 385415.

13. _ Macdonald-koornwinder moments and the two-species exclusion process, arXiv preprint arXiv:1505.00843 (2015). 
14. N. Crampe, K. Mallick, E. Ragoucy, and M. Vanicat, Open two-species exclusion processes with integrable boundaries, arXiv preprint arXiv:1412.5939 (2014).

15. N. Crampe, E. Ragoucy, and M. Vanicat, Integrable approach to simple exclusion processes with boundaries. review and progress, Journal of Statistical Mechanics: Theory and Experiment 2014 (2014), no. 11, P11032.

16. B. Derrida, An exactly soluble non-equilibrium system: the asymmetric simple exclusion process, Physics Reports 301 (1998), no. 1, 65-83.

17. _ Non-equilibrium steady states: fluctuations and large deviations of the density and of the current, Journal of Statistical Mechanics: Theory and Experiment 2007 (2007), no. 07, P07023.

18. B. Derrida, M. R. Evans, V. Hakim, and V. Pasquier, Exact solution of a $1 d$ asymmetric exclusion model using a matrix formulation, Journal of Physics A: Mathematical and General 26 (1993), no. 7, 1493.

19. P. Di Francesco and P. Zinn-Justin, Around the razumov-stroganov conjecture: proof of a multi-parameter sum rule, JOURNAL OF COMBINATORICS 12 (2005), no. 1, R6.

20. A. Doikou, S. Evangelisti, G. Feverati, and N. Karaiskos, Introduction to quantum integrability, International Journal of Modern Physics A 25 (2010), no. 17, 3307-3351.

21. E. Duchi and G. Schaeffer, A combinatorial approach to jumping particles, Journal of Combinatorial Theory, Series A 110 (2005), no. 1, 1-29.

22. G. Gasper and M. Rahman, Basic hypergeometric series, vol. 96, Cambridge university press, 2004.

23. J. E. Humphreys, Reflection groups and coxeter groups, vol. 29, Cambridge university press, 1992.

24. M. Kasatani, Boundary quantum knizhnik-zamolodchikov equation, New trends in quantum integrable systems (2009), 157-171.

25. R. Koekoek and R. F. Swarttouw, The askey-scheme of hypergeometric orthogonal polynomials and its q-analogue, arXiv preprint math/9602214 (1996).

26. T. H. Koornwinder, Askey-wilson polynomials for root systems of type bc, Contemp. Math 138 (1992), 189-204.

27. A. Lazarescu and V. Pasquier, Bethe ansatz and q-operator for the open asep, Journal of Physics A: Mathematical and Theoretical 47 (2014), no. 29, 295202.

28. G. Lusztig, Affine hecke algebras and their graded version, Journal of the American Mathematical Society (1989), 599-635.

29. C. T. MacDonald, J. H. Gibbs, and A. C. Pipkin, Kinetics of biopolymerization on nucleic acid templates, Biopolymers 6 (1968), no. 1, 1-25.

30. K. Mimachi, A duality of macdonald-koornwinder polynomials and its application to integral representations, Duke Mathematical Journal 107 (2001), no. 2, 265-281.

31. M. Noumi, Macdonald-koornwinder polynomials and affine hecke rings, Surikaisekikenkyusho kokyuroku 919 (1995), 44-55.

32. S. Sahi, Nonsymmetric koornwinder polynomials and duality, Annals of mathematics 150 (1999), 267-282.

33. S. Sandow, Partially asymmetric exclusion process with open boundaries, Physical Review E 50 (1994), no. 4, 2660. 
34. K. Shigechi, Laurent polynomial solutions of the boundary quantum knizhnikzamolodchikov equation, arXiv preprint arXiv:1412.7797 (2014).

35. E.K. Sklyanin, Boundary conditions for integrable quantum systems, Journal of Physics A: Mathematical and General 21 (1988), no. 10, 2375.

36. F. Spitzer, Interaction of markov processes, Advances in Mathematics 5 (1970), no. 2, 246-290.

37. J. V. Stokman, Koornwinder polynomials and affine hecke algebras, International Mathematics Research Notices 2000 (2000), no. 19, 1005-1042.

38. J. V. Stokman and B. Vlaar, Koornwinder polynomials and the xxz spin chain, Journal of Approximation Theory (2014).

39. M. Uchiyama, Two-species asymmetric simple exclusion process with open boundaries, Chaos, Solitons \& Fractals 35 (2008), no. 2, 398-407.

40. M. Uchiyama, T. Sasamoto, and M. Wadati, Asymmetric simple exclusion process with open boundaries and askey-wilson polynomials, Journal of Physics A: Mathematical and General 37 (2004), no. 18, 4985.

LPtM, Université de Cergy-Pontoise (CNRS UMr 8089), CergyPontoise Cedex, France.

E-mail address: luigi.cantini@u-cergy.fr 